\title{
Modeling of Beta Conductivity in Tritiated Amorphous Silicon
}

by

\author{
Stefan Costea
}

A thesis submitted in conformity with the requirements

for the degree of Master of Applied Science

Graduate Department of Electrical and Computer Engineering University of Toronto

๑ Copyright by Stefan Costea (2000) 
National Library

of Canada

Acquisitions and Bibliographic Services

395 Wellington Street Ottawa ON K1A ON4 Canada
Bibliothèque nationale du Canada

Acquisitions et services bibliographiques

395, rue Wellington

Ottawa ON K1A ON4

Canada
The author has granted a nonexclusive licence allowing the National Library of Canada to reproduce, loan, distribute or sell copies of this thesis in microform, paper or electronic formats.
L'auteur a accordé une licence non exclusive permettant à la Bibliothèque nationale du Canada de reproduire, prêter, distribuer ou vendre des copies de cette thèse sous la forme de microfiche/film, de reproduction sur papier ou sur format électronique.

L'auteur conserve la propriété du droit d'auteur qui protège cette thèse. $\mathrm{Ni}$ la thèse ni des extraits substantiels de celle-ci ne doivent être imprimés ou autrement reproduits sans son autorisation. 
"Modeling of Beta Conductivity in Tritiated Amorphous Silicon"

Degr:ee of Master of Applied Science

Graduate Department of Electrical and Computer Engineering

University of Toronto

2000

\begin{abstract}
For the first time a model was developed to explain the time evolution of the electrical conductivity in tritiated amorphous silicon samples using data obtained earlier by Mr. Tome Kosteski and Dr. Franco Gaspari.

The model takes into account thermally activated conductivity in the extended states, hopping conductivity in the conduction band tail localized states and beta conductivity in the extended states. The variable parameters are neutral and positively charged dangling bond concentrations, the quasi-Fermi energy for electrons and the hopping distance.

Results show that the main factor affecting the conductivity is the concentration of neutral dangling bonds. The time evolution of the total dangling bond concentration is in agreement with the tritium nuclear decay process. The values found for the Fermi energy and for the hopping distance are in agreement with the literature and support the time evolution of the dangling bond concentration.
\end{abstract}




\section{Acknowledgements}

I would like to thank Professor Stefan Zukotynski for his time and guidance.

I'd like to thank Dr. Franco Gaspari for his advice and for producing and making available the experimental results, which this thesis is based on. I thank Mr. Tome Kosteski for his friendship and for his work in depositing and measuring the sample.

The University of Toronto Open Fellowship is gratefully acknowledged. 


\section{Table of Contents}

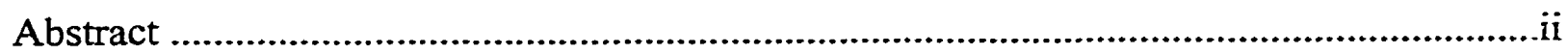

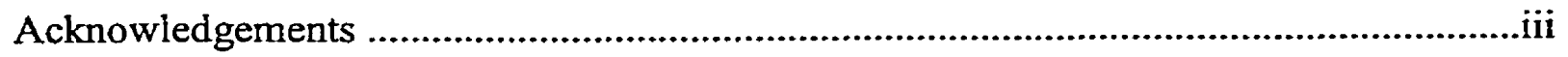

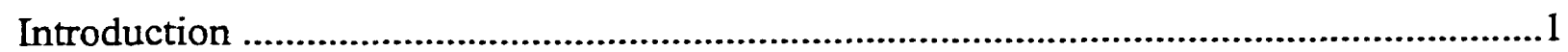

Chapter I Amorphous Silicon .....................................................................

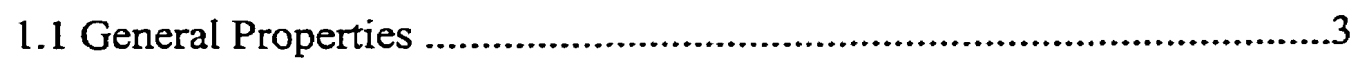

1.2 Dangling Bonds in Amorphous Silicon ......................................16

1.3 Conduction Processes in Amorphous Silicon .................................19

Chapter II $\quad$ Tritiated Amorphous Silicon ................................................................23

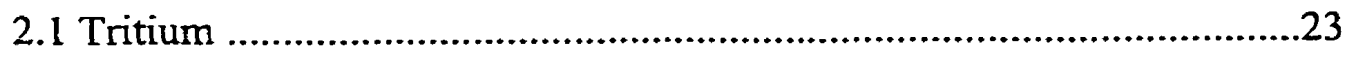

2.2 Tritiated Amorphous Silicon Deposition ......................................24

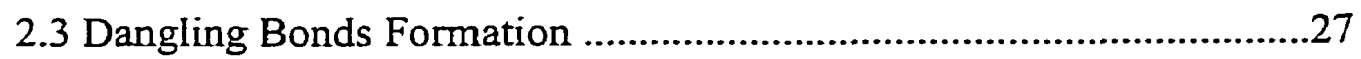

Chapter III Conductivity in Tritiated Amorphous Silicon ........................................30

3.1 Thermally Activated Conductivity .............................................30

3.2 Hopping Conductivity in the Localized States ...............................31

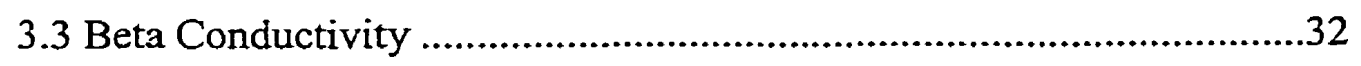

3.4 Modeling the Electrical Conductivity ...........................................36

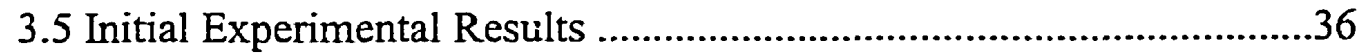

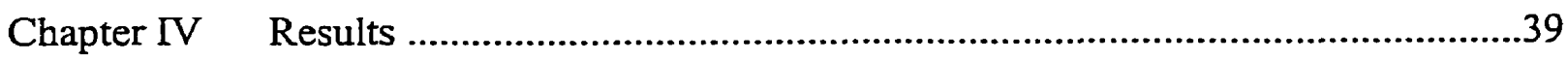

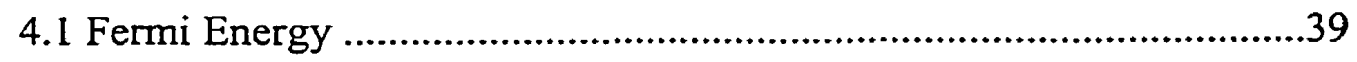

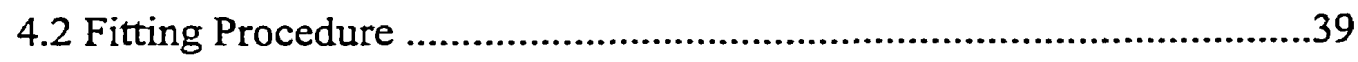

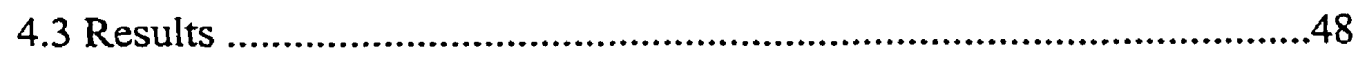

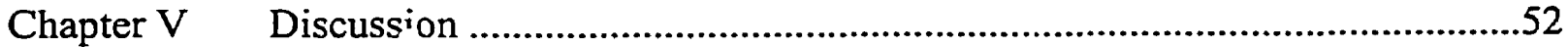




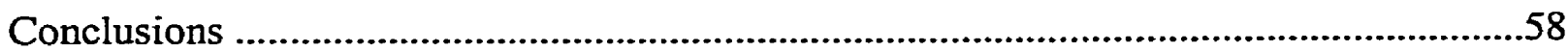

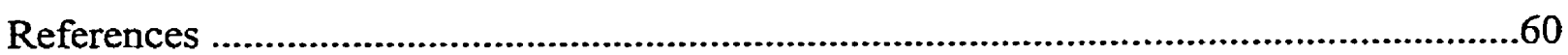




\section{Introduction}

Hydrogenated amorphous silicon has been studied intensively, over the last twenty years, since the introduction of the plasma deposition technique. When prepared by conventional evaporation or sputtering, amorphous silicon contains a large density of defects. Using plasma enhanced chemical vapor deposition (PECVD) significantly reduces the defect density, mainly due to the incorporation of bydrogen atoms.

The incorporation of tritium in amorphous silicon allows a different approach in the study of disorder in amorphous silicon. The decay of tritium is a unique source of disorder in the amorphous silicon network. The decaying bonded tritium atoms create dangling bonds at the tritium-silicon bonds sites.

The purpose of this thesis is to confirm for the first time the direct relation between the nuclear decay process of tritium and the dangling bond formation, which was only indirectly proven before. The experimental data used in this thesis are the electrical conductivity vs. temperature for a tritiated amorphous silicon sample, at different times after deposition obtained by Mr. Tome Kosteski, and Dr. Franco Gaspari on samples prepared by Mr. Tome Kosteski at the Ontario Hydro Tritium Laboratory. A theoretical analysis of this experimental data is the subject of the present thesis.

Chapter I describes the structure and the main properties of amorphous silicon, emphasizing the main electrical transport processes. Chapter II introduces tritiated amorphous silicon and its special properties associated with the tritium decay process. 
Chapter III discusses the electrical conductivity in amorphous silicon and presents the experimental results. Chapter IV describes the fitting procedure and presents the results. A discussion of the results forms chapter $V$. 


\section{Chapter I}

\section{Amorphous Silicon}

\subsection{General Properties}

The development of a new material with useful technological applications is relatively rare. This is because such a material must have either electronic properties or material attributes that cannot be provided by other material. One such relatively new material is amorphous silicon (and its alloys), with increasing use in photovoltaic solar cells and in large area arrays of electronic devices.

Much of the current research into amorphous semiconductors is directed towards an understanding of how the structural disorder leads to their unique properties. Amorphous silicon was first regaided as a derivative of crystalline silicon, with a degradation of the electronic properties due to the disorder. The interest in this material comes from the realization that this is not an accurate view. The disordered atomic structure results in energy states characteristics that are unique to amorphous materials and which are reflected in the optical and electrical properties of these materials.

The study of amorphous silicon progressed rapidly after the discovery of substitutional doping by Spear and Le Comber in 1975, which attracted much attention to the material. A second historically important development was the introduction of the plasiña assisted deposition technique, which uses silane $\left(\mathrm{SiH}_{4}\right)$ decomposed in a $\mathrm{RF}$ or dc glow discharge to form the material. When prepared by conventional evaporation or sputtering, amorphous silicon contains a large density of defects and microvoids, and is 
not reproducible. Using plasma enhanced chemical vapor deposition (PECVD) significantly reduces the defect density. It was quickly discovered that an important component of the PECVD material is the hydrogen, which is responsible for suppressing defects. The incorporated hydrogen atoms supply the covalent bonds needed by the silicon lattice. Of interest is the range of hydrogen content associated with good electronic properties. The indication is that the range $5-12 \%$ in the material is favored and it is worth noting that the hydrogen content for best results reported by a number of groups has shifted downward with the time. This is likely related to improvements in the deposition processes that have been developed. The material structure is better and less hydrogen is needed to reach good properties.

The lack of long range order in the atomic structure is the main feature, which distinguishes amorphous materials from their crystalline counterparts. This is of particular interest in semiconductors, because the periodicity of the atomic structure is central to the theory of crystalline semiconductors. Bloch's theorem is a direct consequence of the periodicity and describes the electrons and holes by wave functions, which are extended in space with quantum states defined by the crystal momentum. The theory of lattice vibrations has similar basis in the lattice symmetry. The absence of an ordered atomic structure in amorphous semiconductors requires a different theoretical approach. The description of these materials is developed instead from the chemical bonding between the atoms, with emphasis on the short range bonding interactions rather than the long-range order.

Although the crystalline and amorphous silicon have similar covalent atomic bonds, the disorder represented by deviations in the bond length and bond angles 
broadens the distribution of electron states and causes carrier localization as well as strong scattering. Structural defects such, as broken bonds, give rise to electronic states which lie in the band gap. There are also new phenomena due to the emphasis on the local chemical bonds rather than the long-range translational symmetry [1].

Disorder is often described with the help of the atom pair distribution function, which is the probability of finding an atom at distance $R$ from another atom. Schematic pair distribution functions for crystalline, amorphous and gaseous phases are illustrated in figure 1.1 .

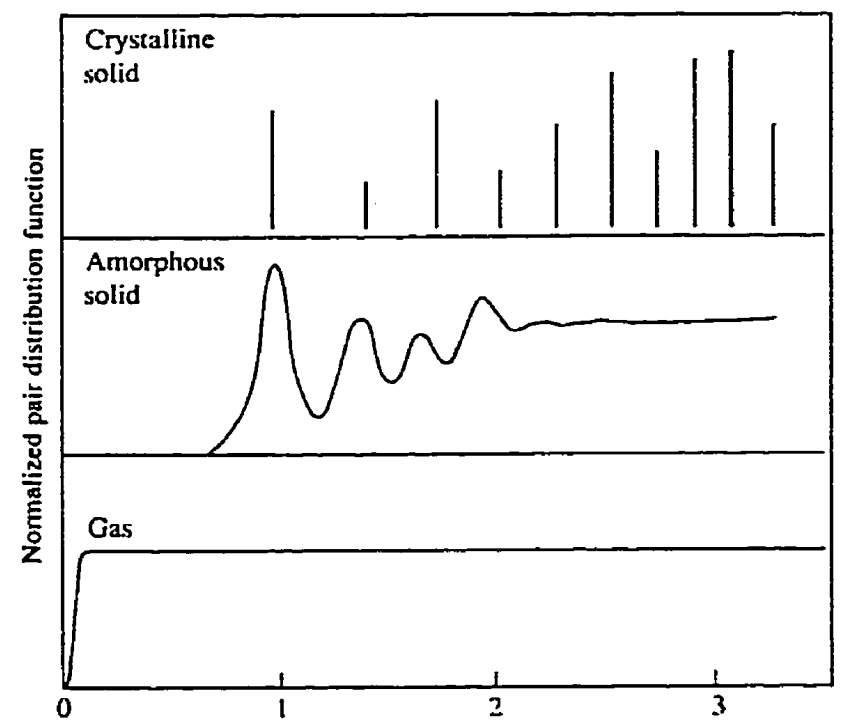

Figure 1.1. Atom pair distribution functions for a crystalline and amorphous solid and a gas, scaled to the average separation of nearest neighbor atoms, $R_{a v}[1]$.

In a dilute gas, the relative position of atoms is random, except at very close spacing, whereas a perfect crystal is completely ordered to large pair distances. An amorphous material has the same short-range order as a crystal, but lacks long-range order. The first 
few nearest neighbors spacing are defined, but the correlation between atom pairs is lost after several inter-atomic distances.

According to the nearly free electron theory, the band gap is a consequence of the periodicity of the crystalline lattice. This suggests that amorphous semiconductors should not exhibit a band gap. However, a band gap can be also justified by the splitting of the bonding and antibonding states in the covalent bond. In this approach, the absence of long-range periodicity is considered a perturbation. It appears that in silicon band formation is influenced more by the short-range configuration of the system, which is similar in crystalline and amorphous material.

So, the three main characteristics of an amorphous semiconductor are:

1. Short range order (similar to the crystalline counterpart),

2. Long range disorder,

3. Coordination defects (unsaturated bonds).

The model for the density of states in the amorphous silicon is presented in figure 1.2.

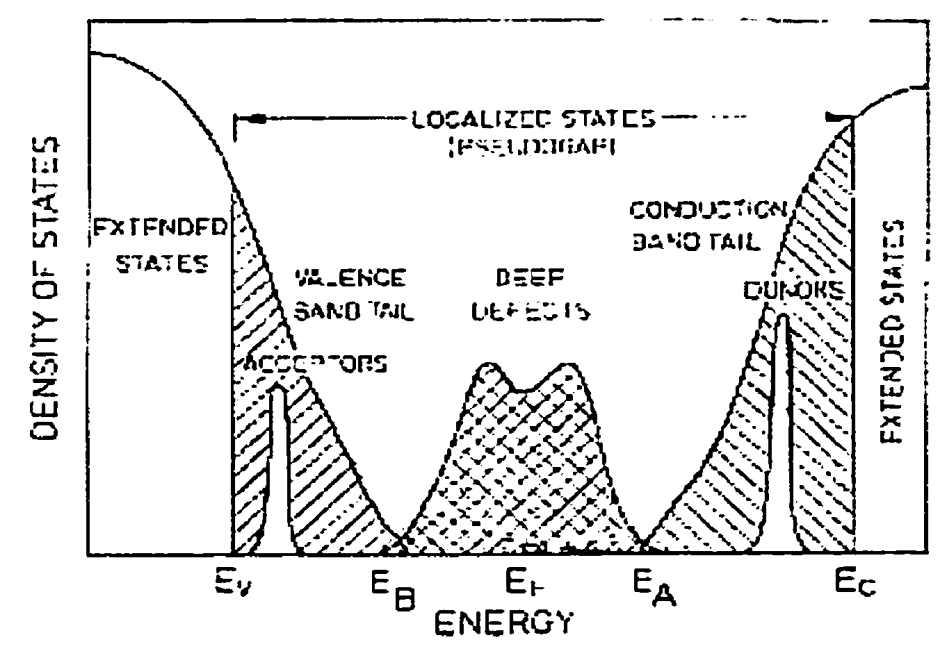

Figure 1.2. Schematic density of states distribution in amorphous silicon [3]. 
The broadened tails of states, which extend into the forbidden gap, replace the band edges. This is mainly due to the variable bond lengths and angles, which represent the long-range structural disorder. The band tail states have an important contribution to the electronic transport. The electronic states deep in the band gap are explained by coordination defects, which determine many electronic properties by controlling trapping and recombination.

The effect of disorder is discussed in [1], where fundamental relations are applied to the amorphous case. The electronic states have wave functions determined by solving the Schrodinger equation, which involves the potential of the lattice $V(r)$ :

$$
-\frac{h^{2}}{2 m} \nabla^{2} \Phi+V(r) \Phi=E \Phi
$$

In a crystalline structure, the lattice is periodic, so the potential $V(r)$ is a periodical function. The solution of Schrodinger's equation for a periodic lattice is given by the Bloch's theorem:

$$
\Phi(r)=\exp (i k \cdot r) U_{k}(r)
$$

where $U_{k}(r)$ is a Bloch function, which reflects the periodicity of the lattice. This periodicity will determine a propagation of the constant phase across the different sites in the crystal and the wave vector, $k$, of the wave function, is conserved. 

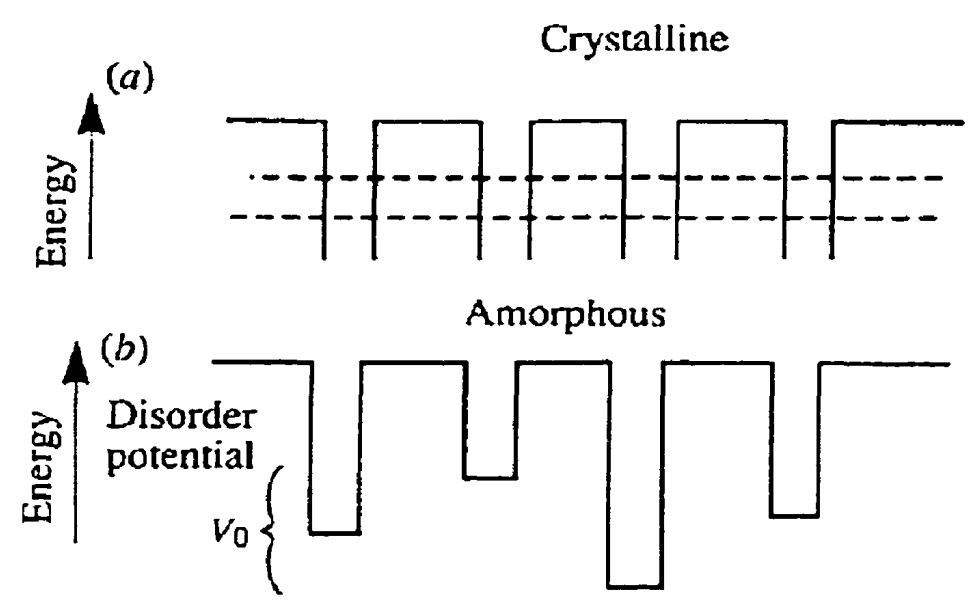

Figure 1.3. The Anderson model of the potential wells for: crystalline lattice, (a), and amorphous network, (b) [1].

The dispersion relations, between the energy $\mathrm{E}$ and the momentum $\mathrm{k}$ lead to energy bands and, further more, to the effective mass and the density of states function.

In an amorphous semiconductor, the lattice is not periodic and the potential $V(r)$ is not periodic. The Anderson model [1] for the potential is presented in figure 1.3, together with the potential in a crystal. This disordered potential will give rise to the scattering of the electron between states. This means that the phase of the wavefunction will not be constant across the crystal (as can be seen in figure 1.4), and the wave vector will not be conserved. In crystalline semiconductors the wave vector is linked to momentum through the dispersion relation. In amorphous materials this simple link does not appear to be available. 


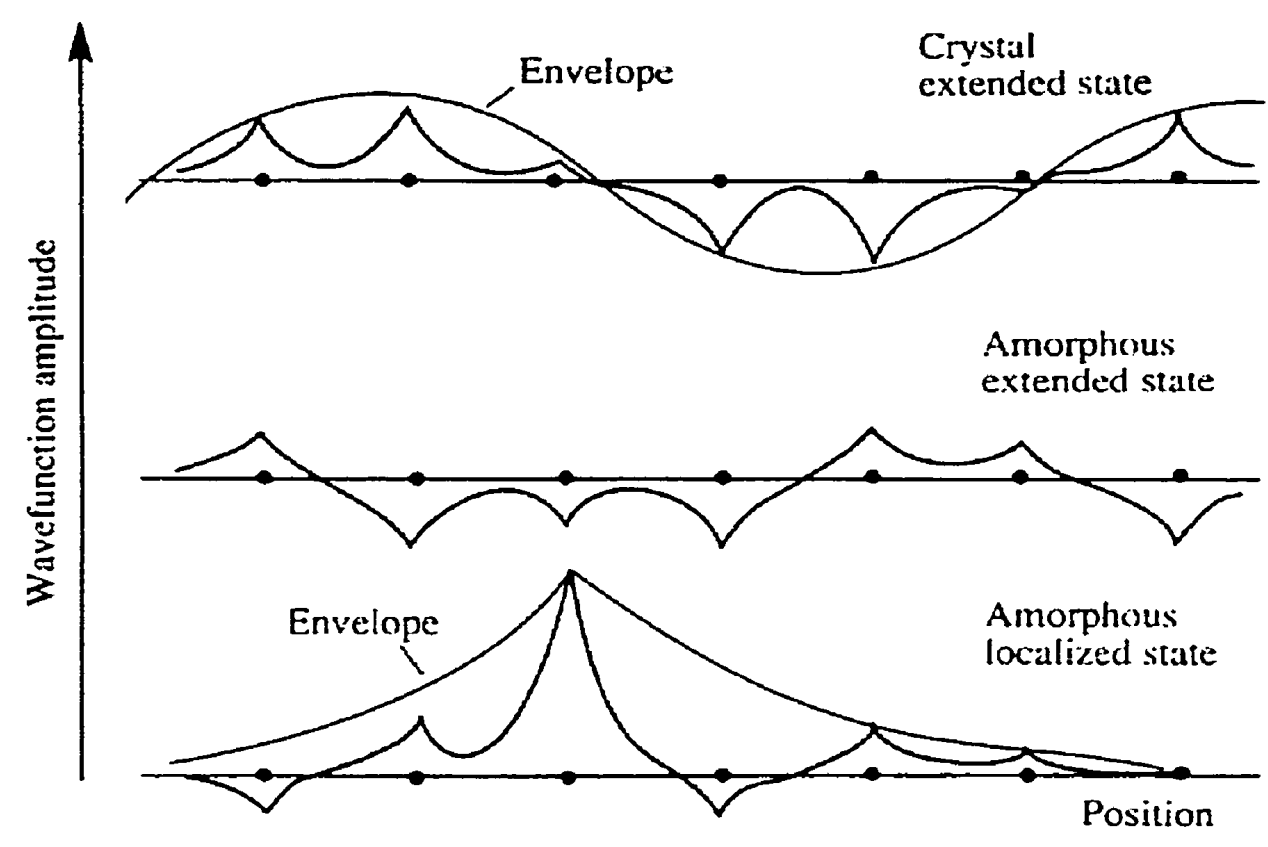

Figure 1.4. The wave functions for extended and localized states in an amorphous material, compared to the extended states in a crystal [1].

Momentum conservation cannot be easily applied to describe the electron states. Therefore, the spatial location of the carrier is more important. Dispersion relations have not been developed to describe energy bands. Energy bands are described in terms of density of states functions, $N(E)$. The density of states distribution for amorphous silicon is presented in figure 1.2. The loss of the phase propagation decreases carrier mobility and determines the localization of the wave function.

The deposition process of hydrogenated amorphous silicon and its alloys is presented in [7]. The details of the process have strong influence on the material properties. Mainly, all the processes developed so far are based on some form of chemical vapor deposition (CVD), in which the amorphous silicon film is produced via 
chemical reactions. Figure 1.5 shows the main events in the history of the amorphous silicon development.

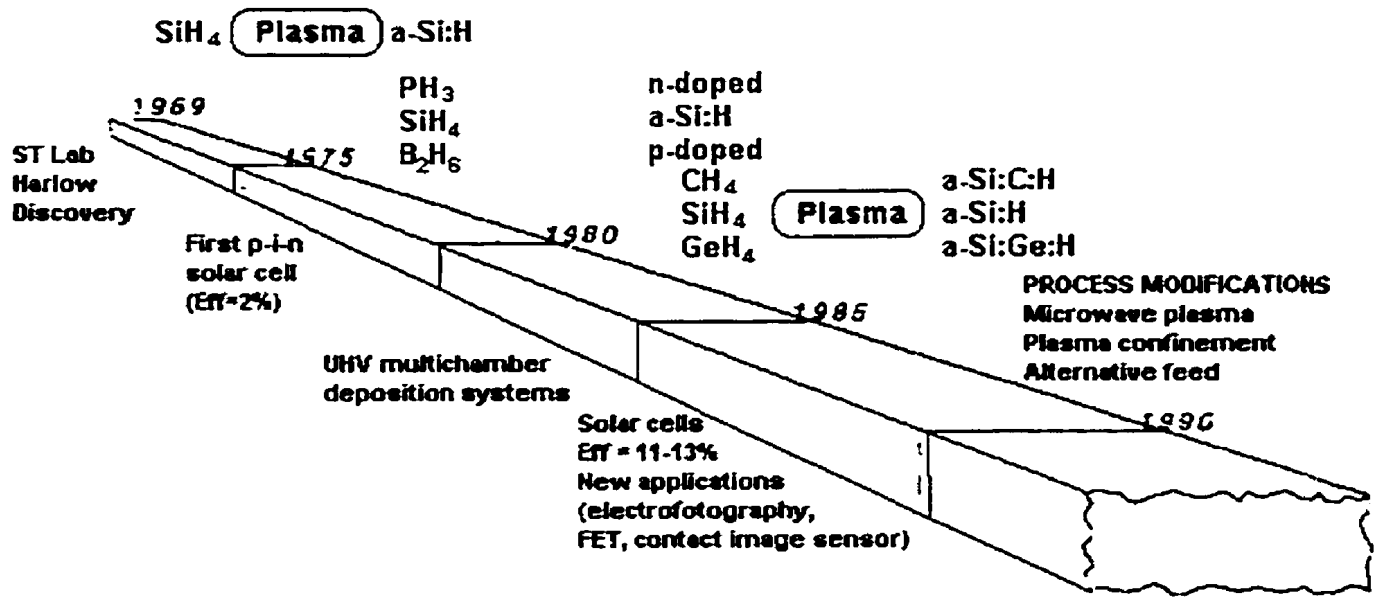

Figure 1.5. History of amorphous silicon: process and technology development [7].

Generally, the deposition process can be expressed as

$$
\mathrm{SiH}_{4} \rightarrow \mathrm{a}-\mathrm{Si}: \mathrm{H}+\mathrm{xH}
$$

The energy is provided to the chemical system is by thermal activation (low pressure CVD), by radiation (photo-CVD) or electrical excitation (plasma enhanced - PECVD). Our interest lies mainly with the PECVD techniques.

A plasma can be defined as a partially ionized, quasi-neutral gas. The gas will become plasma, when energy transfer from an external source increases the number of charged particles. The simplest way to create and sustain plasma for a long time is the electric discharge [7]. In an electrical discharge, the electrons tend to dominate the plasma characteristics. Their collisions can be elastic, when the electron momentum, energy or direction is changed, without affecting the internal energy of the neutral species, or inelastic, when the electron's energy is transferred to the internal energy of other particles, leading to excitation, dissociation or ionization. 
The main plasma reactions are summarized in [7] as:

1. Electron-molecule reactions:

$$
\begin{gathered}
\mathrm{SiH}_{4}+e \rightarrow \mathrm{SiH}_{n}+(4-n) H+e \\
\mathrm{SiH}_{4}+e \rightarrow \mathrm{SiH}_{n}^{+}+(4-n) H+2 e \\
\mathrm{SiH}_{4}+e \rightarrow \mathrm{SiH}_{n}^{-}+(4-n) H
\end{gathered}
$$

2. Neutral-neutral reactions:

$$
\begin{gathered}
\mathrm{SiH}_{4}+\mathrm{H} \rightarrow \mathrm{SiH}_{3}+\mathrm{H}_{2} \\
\mathrm{SiH}_{4}+\mathrm{SiH}_{n} \rightarrow \mathrm{Si}_{2} \mathrm{H}_{4+n} \rightarrow \rightarrow \mathrm{Si}_{n} \mathrm{H}_{m}
\end{gathered}
$$

3. Ion-molecule reactions:

$$
\mathrm{SiH}_{n}^{+}+\mathrm{SiH}_{4} \rightarrow \mathrm{Si}_{2} \mathrm{H}_{m}^{+}+(4+n-m) H
$$

The contribution of the reactions to the growth process depends on the plasma parameters (pressure, power, frequency, and gas composition). In order to have a better control of the deposition, one must consider how the procedure will affect the parameters. A synopsis of the main aspects that should be considered is presented in figure 1.6. 


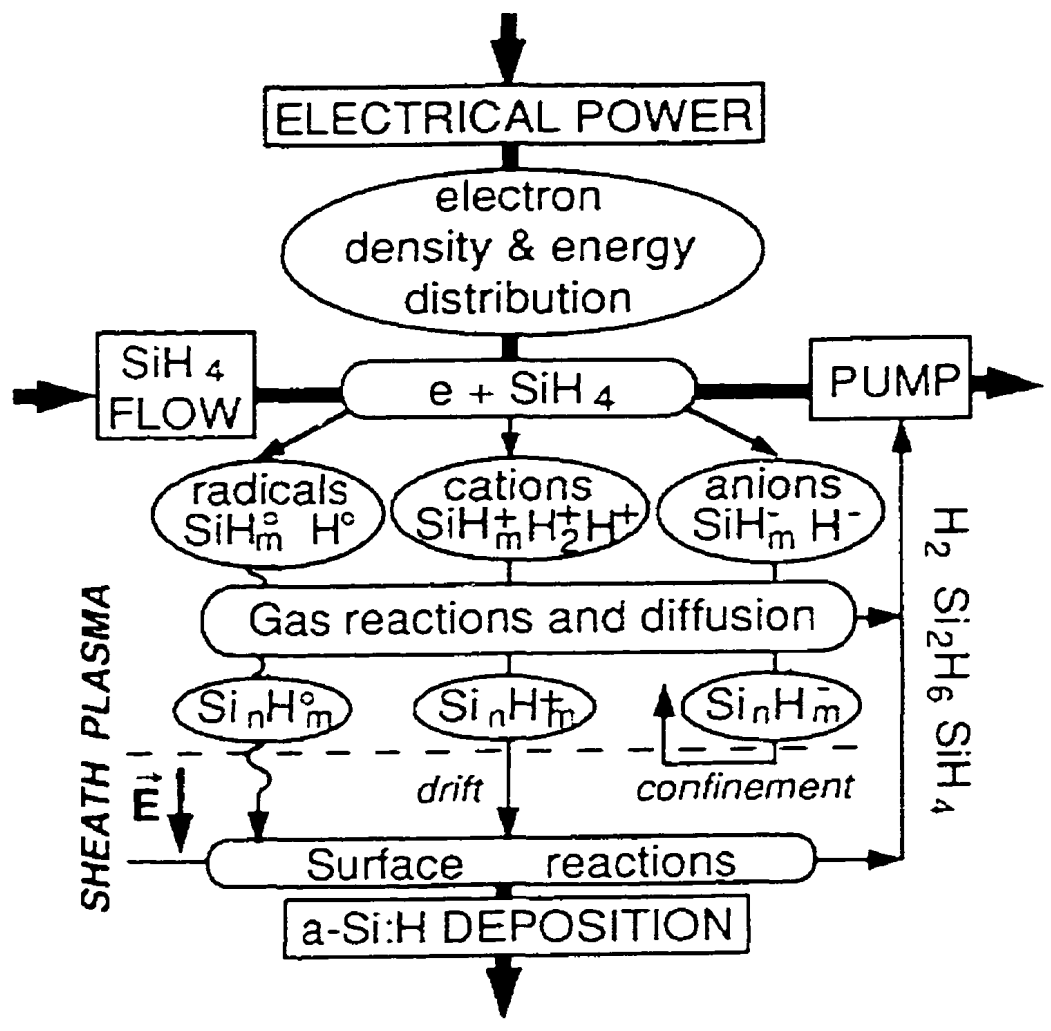

Figure 1.6. Synopsis of the energy and material balance and the reactions in the a-Si:H PECVD process, from $\mathrm{SiH}_{4}[7]$.

As is presented in [1] and [2], the hydrogen has its most important role in maintaining the stability of the amorphous silicon network. The hydrogen and the silicon have different bonding properties, when included in the a-Si:H network. The silicon bonds are rigid and highly strained. After the deposition there is no relaxation because Si atoms do not diffuse. On the other hand, the hydrogen bonds are not rigid and when the bond is broken the atoms can diffuse through the material. The way the hydrogen will bond in a-Si:H network is very sensitive to the deposition conditions and its diffusion affects the electronic properties of the material [2]. 
The stable bonding configurations are $\mathrm{Si}-\mathrm{H}$ and the normal (unstrained) $\mathrm{Si}-\mathrm{Si}$ bonds. The chemical potential of the hydrogen is high enough that the presence of an hydrogen atom will break strained (weak) Si - Si bonds. Therefore, these bonds are easily canceled, and new ones are formed: $\mathrm{Si}-\mathrm{H}$ or stronger (normal) $\mathrm{Si}-\mathrm{Si}$ bonds. These processes can happen throughout the material, due to the diffusion of the hydrogen, and a more ordered network replaces the highly disorder one from before. The process will occur when the deposition is performed at a temperature that allows a hydrogen diffusion process [2].

The presence of bonded hydrogen atoms in the network can be measured by using IR absorption measurements. Because the hydrogen atom is lighter than the silicon atom, the frequencies of the hydrogen vibrational modes are higher than the silicon frequencies. Figure 1.7 presents the normal modes of vibration of the $\mathrm{Si}-\mathrm{H}$ bonds.

The effect of the hydrogen dilution in the deposition process is explained in [7]. The hydrogen radical $\left(\mathrm{H}^{+}\right)$can contribute to the dissociation of the silane molecule by forming a hydrogen molecule and a silane radical (equation 1.7). The silane radicals tend to polymerize, but hydrogen dilution will reduce the probability of these reactions by rehydrogenating the species.

If the pressure is low hydrogen has an almost free path to the surface. This will lead to higher hydrogen content in the film. If the number of hydrogen atoms that are coming to the surface exceeds the numbers of the silane radicals, hydrogen will saturate the coordination defects at the surface. If the ratio of hydrogen atoms over silane radicals is too large, the silane molecules are formed again, and the film growth is slowed or even reversed (etching process). 


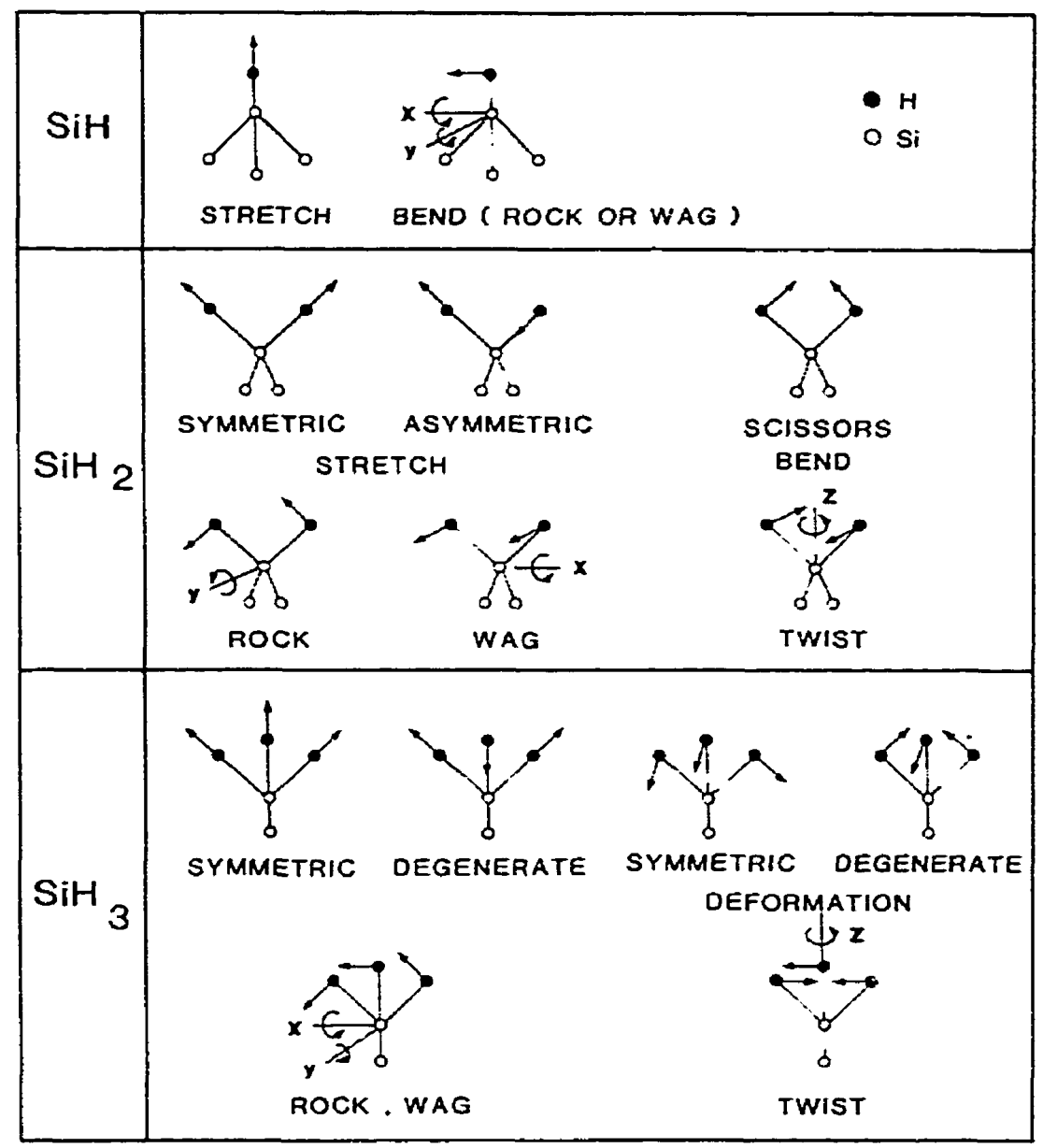

Figure 1.7. The vibrational modes for $\mathrm{Si}-\mathrm{H}$ configurations [2].

The material properties of hydrogenated amorphous silicon have been extensively studied and reviewed. They are unified in Table 1 [4], which characterizes device-quality, undoped a-Si:H deposited by glow-discharge decomposition of silane, with a band gap between 1.7 and $1.8 \mathrm{eV}$, and a hydrogen content of about $10 \%$.

Until the substitutional doping (n-type and p-type) in glow discharge amorphous silicon had been demonstrated, by Spear and LeComber in 1976, it was believed that amorphous semiconductors could not be doped, because the coordination requirements could be satisfied by neighboring atoms. It was subsequently shown that the room 
temperature dark conductivity could be changed by several orders of magnitude with the addition of $\mathrm{P}$ or $\mathrm{B}$ to the amorphous silicon prepared by glow discharge. [2]

The mechanism of doping in amorphous semiconductor is slightly different from that in crystalline material. The process changes the gap state occupancy. The additionai carriers occupy empty gap states near the Fermi level, moving it towards the band edges. Only materials prepared by glow discharge have enough density of gap states to present sensitivity to doping [5].

Introducing well-determined flows of phosphine $\left(\mathrm{PH}_{3}\right)$ or diborane $\left(\mathrm{B}_{2} \mathrm{H}_{6}\right)$ into the silane stream leads to gas phase doping. The gaseous doping ratio (ratio of the number of dopant molecules and silane molecules) is controlled by mixing during the deposition, for ratios higher of $3 \times 10^{-4}$, or by premixing in a reservoir, for values as low as $10^{-6}[5]$.

Ion implantation is Another doping technique. It can be used to control the electrical properties, in the same way the gaseous phase doping is used, by with less efficiency [5].

If the doping process uses both donors and acceptors, the effect of the high defect density in the material can be compensated. One of the properties that are most influenced by the density of defect states is the luminescence. It has been demonstrated that if the luminescence intensity has low values due to the mid-gap defects, then by compensation, luminescence will be restored close to the value for intrinsic material. This process has been explained with reference to the creation of radiative recombination centers. The luminescence is shifted from that for undoped, defect free, material as extra non-radiative recombination centers are created by the compensation [2]. 
Table 1. Typical Room Temperature CEaracteristics of Device-Quality, Undoped a-Si:H [4].

\begin{tabular}{|c|c|c|}
\hline PROPERTY & TYPICAL VALUES & UNITS \\
\hline Optical bandgap, $\mathrm{E}_{\mathrm{g}}$ & $1.7-1.8$ & $\mathrm{eV}$ \\
\hline Hydrogen content $\mathrm{C}_{\mathrm{H}}$ & $8-15$ & at $\%$ \\
\hline Refraction index, $\mathrm{n}$, at $600 \mathrm{~nm}$ & 4.3 & \\
\hline Electron drift mobility, $\mu_{\mathrm{e}}$ & $\geq 1$ & $\mathrm{~cm}^{2} \mathrm{~V}^{-1} \mathrm{~s}^{-1}$ \\
\hline Hole drift mobility, $\mu_{h}$ & $\geq 0.008$ & $\mathrm{~cm}^{2} \mathrm{~V}^{-1} \mathrm{~s}^{-1}$ \\
\hline Electron lifetime, $\tau_{e}$ & $\geq 2 \times 10^{-7}$ & s \\
\hline Hole lifetime, $\tau_{h}$ & $\geq 10^{-6}$ & $\mathbf{s}$ \\
\hline$\mu \tau$ (electron) & $\geq 2 \times 10^{-7}$ & $\mathrm{~cm}^{2} \mathrm{~V}^{-1}$ \\
\hline$\mu \tau$ (hole) & $\geq 10^{-8}$ & $\mathrm{~cm}^{2} \mathrm{~V}^{-1}$ \\
\hline Hole diffusion length, $L_{h}$ & 0.3 & $\mu \mathrm{m}$ \\
\hline Photoconductivity, $\sigma_{\mathrm{p}}$ & $5 \times 10^{-5}-10^{-4}$ & $\mathrm{~S} \mathrm{~cm}^{-1}$ \\
\hline Dark conductivity, $\sigma_{\mathrm{d}}$ & $10^{-11}-10^{-10}$ & $\mathrm{~S} \mathrm{~cm}^{-1}$ \\
\hline$\sigma_{\mathrm{d}}$ activation energy, $\mathrm{E}_{\mathrm{a}}$ & $0.7-0.9$ & $\mathrm{eV}$ \\
\hline Photosensitivity, $\frac{\sigma_{p}}{\sigma_{d}}$ & $\geq 1 \times 10^{6}$ & \\
\hline $\begin{array}{l}\text { Valence band tail extension } \\
\text { into the band-gap, } E_{\mathrm{u}}\end{array}$ & $42-50$ & $\mathrm{meV}$ \\
\hline $\begin{array}{c}\text { Conduction band tail extension } \\
\text { into the band-gap }\end{array}$ & 25 & $\mathrm{meV}$ \\
\hline Density of states at $E_{f}$ & $5 \times 70^{14}$ & $\mathrm{eV}^{-1} \mathrm{~cm}^{-3}$ \\
\hline
\end{tabular}

\subsection{Dangling Bonds in Amorphous Silicon}

The high density of defects in pure amorphous silicon motivated the search for a method to reduce their effects. Hydrogenated material is a big improvement. Although 
the defect density is greatly reduced, the remaining defects still control many electronic properties. [1]

Considering their influence on the material properties, the defects can be described with reference to three main areas of consideration [1]:

- the defect reactions, which describe how the defect density is determined by the growth process and the treatment after;

- the atomic structure and bonding, which determine the electronic state of the defect;

- the energy levels of their different charge states, which determine whether they act as traps or recombination centers.

The defect in an amorphous network cannot be defined the same way as in the crystalline network. In a crystal, any departure from the perfect lattice is considered a defect: point defect (vacancy or interstitial), extended defect (dislocation or stacking fault), impurities, etc. An ideal amorphous network has all atoms fully bonded. Therefore, in the amorphous material disorder must generate situations that would be considered defects in a crystal. In a-Si:H, the ideal situation is with all the silicon atoms four-fold coordinated and all the hydrogen singly coordinated [1]. The only way of having something that will not satisfy this ideality criterion is by having a coordination defect. If the coordination of the atom is different from the ideal one ( 4 for silicon), the atom will have an unpaired electron, when in neutral state. This kind of defect will have a paramagnetic spin. The presence of a paramagnetic spin would thus identify electronic states that are not present in an ideal network. This kind of defect is called dangling bond. If the bond is broken, and both electrons of the covalent bond are lost, the remaining 
configuration will have a positive net charge and a positively charged dangling bond (D ${ }^{+}$) is created. If this state captures a free electron, the neutrality is restored, and the new state is called a neutral dangling bond $\left(\mathrm{D}^{0}\right)$, and is a stable configuration. Furthermore, if another free electron is captured, a negatively charged dangling bond ( $\mathrm{D}^{-}$) appears, with a lower probability, though.

In hydrogenated material, there are also other configurations that differ from the normal amorphous network, but the electronic states formed will be very similar to those of the bulk [1].

Four generic types of defects exist in hydrogenated amorphous silicon based on the difference $\Delta z$ between the coordination numbers of the defect and of the ideal case. In Table 2 are presented the four types [1].

Table 1.2 Possible types of defects in a-Si:H

\begin{tabular}{|c|c|c|c|}
\hline$\Delta \mathbf{z}$ & Defect & States in the gap & $\begin{array}{c}\text { Magnetic properties in the } \\
\text { neutral state }\end{array}$ \\
\hline-2 & $\begin{array}{c}\text { Divacancy } \\
\text { (two-fold coordinated } \\
\text { silicon) }\end{array}$ & 4 & Diamagnetic \\
\hline-1 & Dangling bond & 2 & Paramagnetic \\
\hline 0 & $\begin{array}{c}\text { Hydrogenated void } \\
\text { (weak Si - Si bond) }\end{array}$ & $\begin{array}{c}\text { (only in case of } \\
\text { large bond } \\
\text { distortions) }\end{array}$ & Diamagnetic \\
\hline 1 & The floating bond & 2 & Paramagnetic \\
\hline
\end{tabular}

The dangling bond density is very sensitive to the details of the deposition process. This is one of the main reasons to use hydrogenated material: the hydrogen causes a very important reduction of the dangling bond density. However, even in the 
hydrogenated material, the density of dangling bonds can vary by several orders of magnitude when the deposition conditions are changed.

The dangling bond states act as recombination or trapping centers. The most probable transitions involving the dangling bonds are between the defect states and the extended states (valence and conduction band). If we have the three types of dangling bonds, positively charged $\left(\mathrm{T}^{+}\right)$, negatively charged $\left(\mathrm{T}^{-}\right)$and neutral $\left(\mathrm{T}^{0}\right)$, the recombination or trapping reactions are:

$$
\begin{aligned}
& \mathrm{T}^{+}+\mathrm{e} \leftrightarrow \mathrm{T}^{0} \\
& \mathrm{~T}^{0}+\mathrm{e} \leftrightarrow \mathrm{T}^{-}
\end{aligned}
$$

If the density of dangling bonds is large, another reaction can happen, although with small probability:

$$
\mathrm{T}^{+}+\mathrm{T}^{-} \leftrightarrow 2 \mathrm{~T}^{0}
$$

The importance of the dangling bonds and the carrier recombination process will be discussed further in chapter 4 .

\subsection{Conduction Processes in Amorphous Silicon}

The mobility edge was defined by Mott and Davis [6] as the energy value that separates the extended states (in conduction and valence bands) from localized states (in the band tails). Therefore, the main conductivity processes in amorphous are identified by whether the contributing carriers are in extended states, localized states or defect states. 
a) Conduction in extended states

In the extended states, the electronic transport occurs via carriers excited beyond the mobility edge into the states at or above $\mathrm{E}_{\mathrm{c}}$ (or below $\mathrm{E}_{\mathrm{v}}$ ). In this case, the conductivity is given by the expression [2]:

$$
\sigma \cong e \int g(E) \mu(E) f(E) d E
$$

where

$\mathrm{g}(\mathrm{E})$ is the density of states at level E;

$\mu(E)$ is the carrier mobility at energy $E$;

$$
f(E)=\frac{1}{1+\exp \left(\frac{E-E_{F}}{k_{B} T}\right)} \text {, is the Fermi-Dirac distribution function. }
$$

Assuming that most of the carriers are within about $k_{B} T$ of the edge $E_{c}$, equation 1.12 can be written as:

$$
\sigma \cong \operatorname{eg}\left(E_{C}\right) \mu_{e x t} k_{B} T \exp \left(-\frac{E_{C}-E_{F}}{k_{B} T}\right)
$$

The value for the extended states mobility $\mu_{\text {ext }}$ can be approximated by the minimum metallic conductivity $\sigma_{\min }$, defined in [6] as the conductivity value at $\mathrm{E}_{\mathrm{C}}$, where the localization occurs. This is supposed to be the smallest value that the conductivity at $\mathrm{T}=0$ can have, for a system in which, at $\mathrm{T}=0$, electron states are occupied up to Fermi energy. If the conductivity is expressed as:

$$
\sigma=\sigma_{\min } \exp \left(-\frac{E_{C}-E_{F}}{k_{B} T}\right)
$$

the extended states mobility $\mu_{\text {ext, }}$ will be defined by equation (1.15) 


$$
\mu_{e x t}=\frac{\sigma_{\min }}{\operatorname{eg}\left(E_{c}\right) k_{B} T}
$$

For an effective density of states at $E_{C}$ of $10^{21} \mathrm{~cm}^{-3} \mathrm{eV}^{-1}$, the mobility is estimated at $\mu_{\mathrm{ext}}=10 \mathrm{~cm}^{2} \mathrm{~s}^{-1} \mathrm{~V}^{-1}[6]$

b) Conduction in the tail states

In the tail states, the carrier transport is mainly a hopping process between the localized states [6]. The conductivity at an arbitrary energy $E=E_{x}$ in the tail states $\left(E_{A}<\right.$

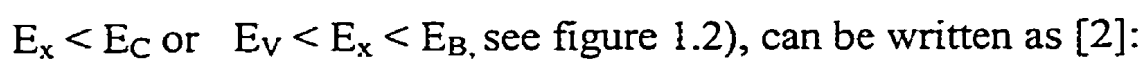

$$
\sigma=e \mu_{\text {hop }} \int_{E_{A}}^{E_{C}} g\left(E_{x}\right) \exp \left(-\frac{E_{x}-E_{F}}{k_{B} T}\right) d E
$$

where $\mu_{\text {hop }}$ is the hopping mobility, and can be expressed by using the Einstein relation

$$
\mu=\frac{e D}{k_{B} T}
$$

by using the expression for diffusion coefficient $D[2]$ :

$$
D=\frac{1}{6} v_{p h} R^{2} \exp \left(-\frac{W_{1}}{k_{B} T}\right)
$$

where

$$
\begin{aligned}
& v_{\mathrm{ph}} \text { is the phonon frequency; } \\
& \mathrm{R} \text { is the hopping distance; }
\end{aligned}
$$

$\mathrm{W}_{\mathrm{l}}$ is the hopping energy, the difference between the energy of the states.

Therefore, the hopping mobility is [2]:

$$
\mu_{\text {hop }}=\frac{1}{6} \nu_{p h}\left(\frac{e R^{2}}{k_{B} T}\right) \exp \left(-\frac{W_{I}}{k_{B} T}\right)
$$

If we consider the energy within $\mathrm{k}_{\mathrm{B}} \mathrm{T}$ of $\mathrm{E}_{\mathrm{A}}$, the conductivity becomes [2]: 


$$
\sigma=e^{2} v_{p h} R^{2} g\left(E_{x}\right) \exp \left(-\frac{E_{x}-E_{F}+W_{1}}{k_{B} T}\right)
$$

c) Conductivity in the band gap

The carrier transport in the band gap is based on hopping between the defect states, which occurs when the material has a high defect density [2]. This process is referred to as variable range hopping. The conductivity associated with variable range hopping is [6]:

$$
\sigma=\sigma_{2} \exp \left(-\frac{B}{T^{1 / 4}}\right)
$$

where $\sigma_{2}=e^{2} g\left(E_{f}\right) r^{2} v_{p h}$, with $r=\left(\frac{3}{2 \pi \alpha_{L} g\left(E_{f}\right) k_{B} T}\right)^{1 / 4}$ and $B=1.66\left(\frac{\alpha_{L}^{3}}{k_{B} g\left(E_{f}\right)}\right)^{1 / 4} \cdot \alpha_{L}$ is the exponent from the overlap integral of the wave functions $e^{-2 \alpha} L^{R}$ (where $R$ is the distance separating the two states).

These are the conduction mechanisms normally associated with amorphous silicon. In the presence of external stimulation, e.g. illumination, redistribution of carriers will occur and modification to the expressions given above must be considered. 


\section{Chapter II}

\section{Tritiated Amorphous Silicon}

Tritiated-hydrogenated amorphous silicon (a-Si:H:T) is a novel thin film material in which tritium is incorporated in the amorphous silicon lattice. The decay process of the tritium will give rise to new phenomena in the material including changes in electrical, optical and structural properties.

\subsection{Tritium}

Hydrogen has two heavy isotopes: deuterium and tritium. Deuterium is stable and has a natural abundance. Tritium is not stable but can be created in nuclear reactions. Urey and Brickwedde discovered tritium in 1931. In 1939, Alvarez and Cornog established that tritium was a radioactive material, and after several years determined an accurate value for the nuclear half-life period by observing tritium removal by surface absorption, isotopic exchange, and diffusion losses [8].

Due to cosmic radiation, a number of atoms in the atmosphere are continuously converted to tritium. The rate of tritium atoms production is $0.25 \pm 0.1 \mathrm{~cm}^{-2} \mathrm{~s}^{-1}$, leading to a concentration in surface water of about $10^{-18}$ tritium nuclei per hydrogen nucleus. This value is called a Tritium Unit (TU) [8].

The nuclear reaction of tritium decay is:

$$
\mathrm{T} \rightarrow{ }^{3} \mathrm{He}^{+}+\mathrm{\beta}^{-}+\bar{v}
$$

Tritium decay produces a helium ion, a beta particle and an antineutrino. The decay has a probability of $1.78 \times 10^{-9} \mathrm{~s}^{-1}$, which means a half-life for tritium of 12.3 
years. The beta particle produced during the tritium decay has a maximum energy of 18.6 $\mathrm{keV}$, and a mean energy is $5.69 \mathrm{keV}$ [8]. Figure 2.1 presents the probability $\mathrm{P}(\mathrm{E}) \mathrm{dE}$ that the energy of a beta particle be in the range of $\mathrm{dE}=112 \mathrm{eV}$ around the value $\mathrm{E}$.

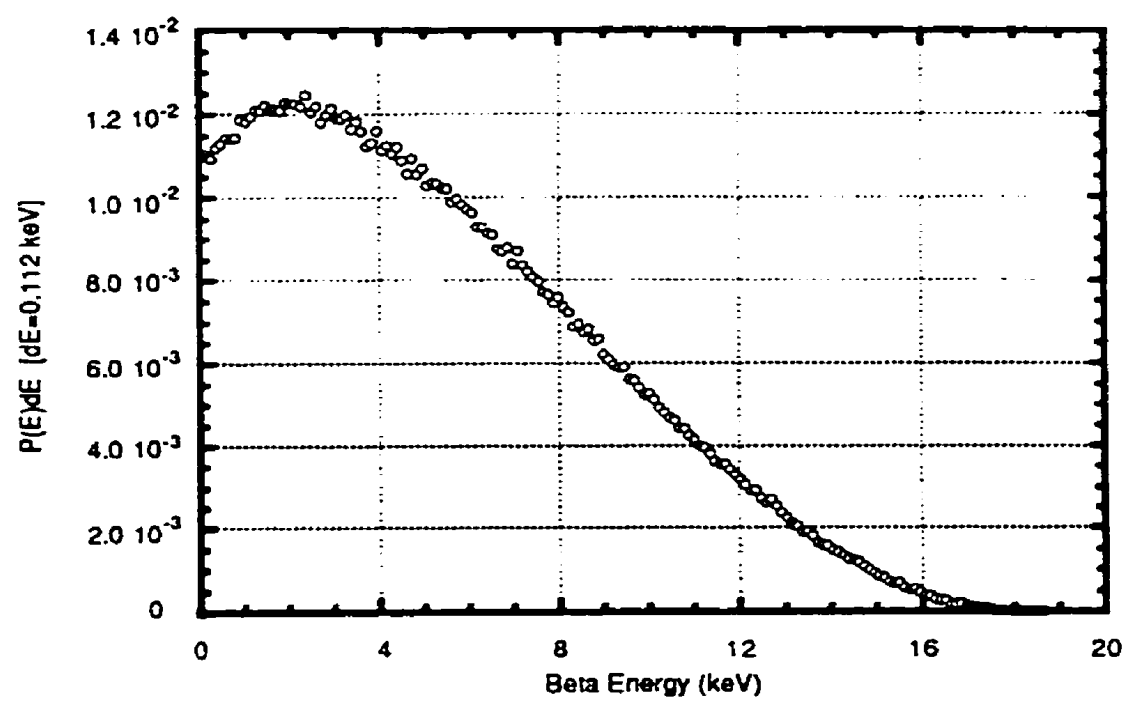

Figure 2.1 Tritium decay beta energy distribution [8].

\subsection{Tritiated Amorphous Silicon Deposition}

The deposition technique used by us for the deposition of tritiated amorphous silicon is dc saddle-field plasma deposition. The samples used in this thesis were deposited by using the deposition system presented schematically in figure 2.2 .

The system consists of a stainless steel chamber with five electrodes: a central mesh anode, two mesh cathodes, a substrate holder at one end, and the chamber flange at the other end [9]. The anode is biased at a dc potential with a typical value around 600V. The two cathodes are at ground potential. The substrate can be floating, biased, or at ground potential, depending on the deposition requirements. A molecular drag pump and a diaphragm pump are used in series to bring the chamber pressure to about 100 mTorr. 
The gases for the deposition are introduced into the chamber through individual mass flow controllers. For tritiated amorphous silicon, only silane is used. For doped material deposition gas mixtures of diborane in silane are used for p-type doping and phosphine in silane for n-type doping. The tritium source is a depleted uranium metal tritide bed. When the uranium bed is heated, tritium escapes the material. The bed temperature controls the partial pressure of the tritium over the bed at equilibrium. The tritium flow into the chamber is regulated by a calibrated pinhole, which assures a determined flow if the tritium pressure over the uranium bed is maintained constant.

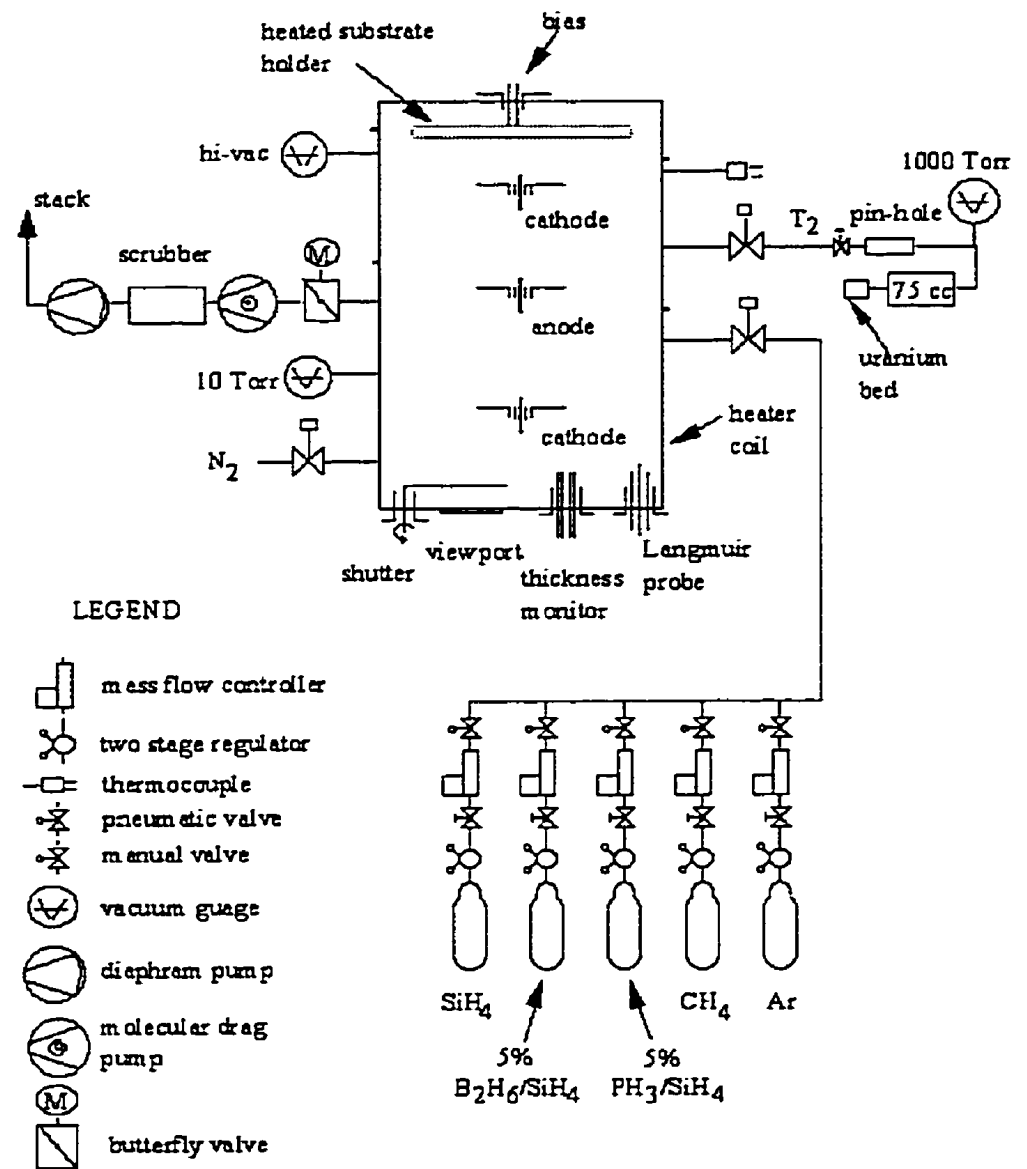

Figure 2.2. Schematic of the dc saddle-field deposition system [9]. 
During depositions that involve tritium flow, the diaphragm pump is disconnected and the exhaust of the molecular drag pump is directed to an $800 \mathrm{~g} \mathrm{Zr}_{2} \mathrm{Fe}$ alloy scavenger bed which irreversibly reacts with silane and stores tritium as a tritide for later removal. The total pressure of the plasma is auto-controlled by a motorized butterfly valve in a feedback loop with a pressure transducer mounted on the chamber.

The three mesh electrodes are placed symmetrically inside the chamber. The electric field created by the anode bias potential accelerates electrons. The anode is semitransparent to the passage of the electrons, which will be decelerated by the electric field between the anode and the other cathode. This process occurs on both sides of the anode, leading to oscillation of the electrons. The low pressure allows a long path for the electrons. The collisions of high energetic electrons with the gas molecules lead to excitation, ionization and dissociation processes. The neutral radical formation is described in equation (1.4) which, for different values of $n$, becomes:

$$
\begin{array}{lll}
\mathrm{n}=0 & \Rightarrow & \mathrm{SiH}_{4}+\mathrm{e}^{-} \rightarrow \mathrm{Si}+2 \mathrm{H}_{2}+\mathrm{e}^{-} \\
\mathrm{n}=1 & \Rightarrow & \mathrm{SiH}_{4}+\mathrm{e}^{-} \rightarrow \mathrm{SiH}+\mathrm{H}_{2}+\mathrm{H}+\mathrm{e}^{-} \\
\mathrm{n}=2 & \Rightarrow & \mathrm{SiH}_{4}+\mathrm{e}^{-} \rightarrow \mathrm{SiH}_{2}+\mathrm{H}_{2}+\mathrm{e}^{-} \\
\mathrm{n}=3 & \Rightarrow & \mathrm{SiH}_{4}+\mathrm{e}^{-} \rightarrow \mathrm{SiH}_{3}+\mathrm{H}+\mathrm{e}^{-}
\end{array}
$$

Equation (1.5) explains the positive ion formation. For $\mathrm{n}$ from 0 to 3 , equation (1.5) can be written:

$$
\begin{array}{lll}
\mathrm{n}=0 & \Rightarrow & \mathrm{SiH}_{4}+\mathrm{e}^{-} \rightarrow \mathrm{Si}^{+}+2 \mathrm{H}_{2}+2 \mathrm{e}^{-} \\
\mathrm{n}=1 & \Rightarrow & \mathrm{SiH}_{4}+\mathrm{e}^{-} \rightarrow \mathrm{SiH}^{+}+\mathrm{H}_{2}+\mathrm{H}+2 \mathrm{e}^{-} \\
\mathrm{n}=2 & \Rightarrow & \mathrm{SiH}_{4}+\mathrm{e}^{-} \rightarrow \mathrm{SiH}_{2}^{+}+\mathrm{H}_{2}+2 \mathrm{e}^{-}
\end{array}
$$


$\mathrm{n}=3 \quad \Rightarrow \quad \mathrm{SiH}_{4}+\mathrm{e}^{-} \rightarrow \mathrm{SiH}_{3}^{+}+\mathrm{H}+2 \mathrm{e}^{-}$

Negative ions are formed as described in equation (1.6), for different values of $\mathrm{n}$ :

$$
\begin{array}{lll}
\mathrm{n}=0 & \Rightarrow & \mathrm{SiH}_{4}+\mathrm{e}^{-} \rightarrow \mathrm{Si}^{-}+2 \mathrm{H}_{2} \\
\mathrm{n}=\mathrm{i} & \Rightarrow & \mathrm{SiH}_{4}+\mathrm{e}^{-} \rightarrow \mathrm{SiH}^{-}+\mathrm{H}_{2}+\mathrm{H} \\
\mathrm{n}=2 & \Rightarrow & \mathrm{SiH}_{4}+\mathrm{e}^{-} \rightarrow \mathrm{SiH}_{2}^{-}+\mathrm{H}_{2} \\
\mathrm{n}=3 & \Rightarrow & \mathrm{SiH}_{4}+\mathrm{e}^{-} \rightarrow \mathrm{SiH}_{3}^{-}+\mathrm{H}
\end{array}
$$

Ionized radicals will be accelerated by the electric field, allowing them to pass the mesh cathode and reach the substrate. The neutral radicals will diffuse in the plasma. Due to the symmetrical configuration of the system, the radical concentration is uniform in the chamber. Tritiated amorphous silicon will be deposited on all the chamber walls, but the deposition on the substrate holder provides bias and temperature control. Depending on the length of deposition, the temperature of the uranium bed may have to be raised, in order to maintain a constant tritium pressure at the input of the pinhole and a constant tritium flow into the chamber.

\subsection{Dangling Bonds Formation}

The tritium decay described by the equation (3.1) represents a permanent transmutation of hydrogen. When the tritium is not bonded the only consequence is beta particle emission and creation and the conversion of tritium in helium. After the incorporation in the amorphous silicon matrix, the physical consequences of the decay process increase, due to its effects on the material. 
In the tritiated amorphous silicon matrix, tritium atoms replace the hydrogen atoms of the conventional hydrogenated amorphous silicon. Each decaying tritium atom will generate a beta particle, an electron with high energy. These particles will interact with the amorphous network and generate over 1000 electron-hole pairs each. Therefore, many free electrons and holes will be present throughout the material, many more than in the normal hydrogenated amorphous silicon lattice. At the same time, the bonded tritium atoms are converted in positively charged helium ions, which will not bond with the silicon atoms. The helium recoil energy is about $3 \mathrm{eV}$. The site where tritium previously bonded will be left empty, and the silicon atom will have an unsatisfied bond. Helium ions have very high ionization energy and will attract both electrons involved in the initial covalent bond between the silicon and the tritium. In this way, the helium ions will be neutralized into helium atoms. In the end, the silicon atom has an unsatisfied bond described as a positively charged dangling bond $\left(\mathrm{D}^{+}\right.$center). With time, the $\mathrm{D}^{+}$center will attract an electron and be converted into a neutral dangling bond ( $\mathrm{D}^{0}$ center). This process has a high probability due to the large number of free electrons present in the material. Figure 2.3 presents schematically the decay process and its direct consequences.

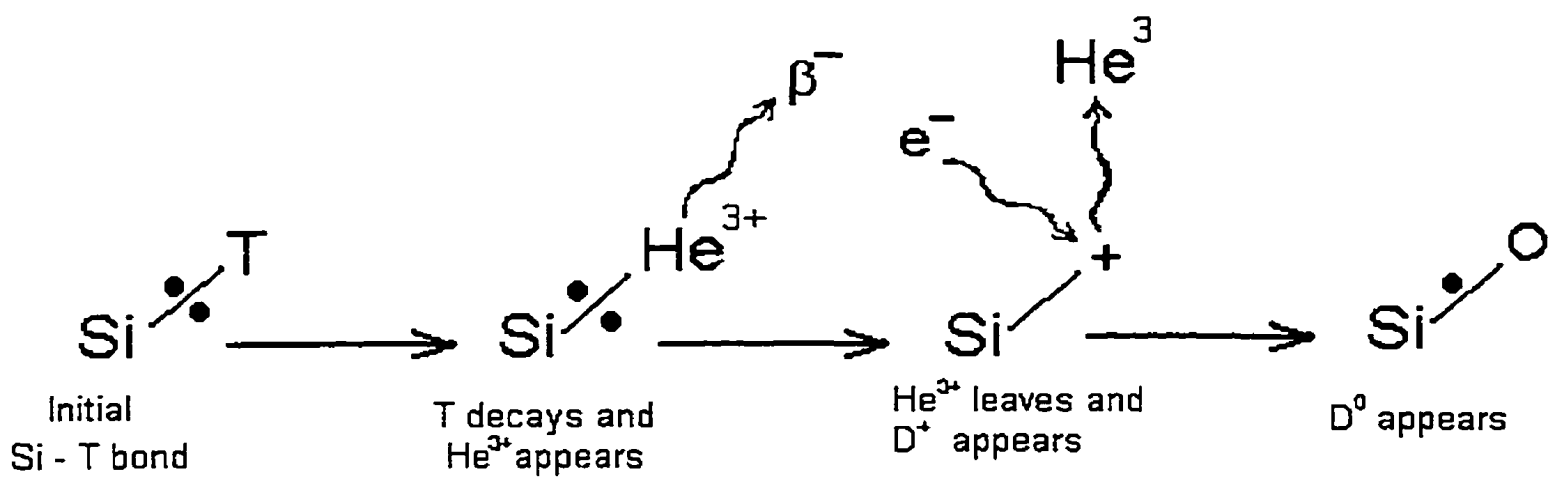

Figure 2.3. The mechanism of tritium decay and dangling bond formation. 
It is expected that, at least initially, a competition between $\mathrm{D}^{+}$and $\mathrm{D}^{0}$ states will occur, with the latter being the dominant one due to the large amount of free electrons available to be attracted by the $\mathrm{D}^{+}$dangling bond. Crandall [10] argues that $\mathrm{D}^{+}$defects, being near the band edges, are readily thermally ionized back to their original charged state, except for a fraction that will stabilize in the neutral state.

Lattice relaxation is necessary for such a stabilization to occur since for the defect to be in the $D^{0}$ state, a $15^{\circ}$ bond-angle change is necessary [10]. The dangling bonds created by tritium decay have enough local energy, due to lattice relaxation, energetic beta particles and the $3 \mathrm{eV}$ helium recoil, to adjust the bond angle and assume the $\mathrm{D}^{0}$ configuration.

Tritium decay is a unique mechanism to create dangling bonds in the material, with two main advantages:

- It can create dangling bonds without affecting the surrounding lattice;

- The rate of the dangling bond formation is accurately determined, as it is the direct consequence of the nuclear decay process.

The process is also of potential technological importance, due to the creation of the electron-hole pairs, without external intervention. Possible applications can be voltaic cells (similar to solar cells) and auto-luminescent devices, utilizing radioactive recombination of the carriers. 


\section{Chapter III}

\section{Conductivity in Tritiated Amorphous Silicon}

Tritiated-hydrogenated amorphous silicon (a-Si:H:T) is a form of hydrogenated amorphous silicon, with tritium atoms replacing some of the hydrogen atoms in the amorphous lattice. Since tritium is an isotope of hydrogen most of the physical properties should be quite similar to those of the pure hydrogenated material. However, due to the beta decay of tritium, some material properties will be different. This thesis considers only the influence of the tritium beta decay process on the electrical conductivity of aSi:H:T.

In a quantitative approach, one should take into account the transport mechanisms that are most likely to be important, in the temperature range being considered. In our case, the temperature range is between 20 and $200{ }^{\circ} \mathrm{C}$.

The transport due to holes is ignored, because of their low mobility.

\subsection{Thermally Activated Conductivity}

The general expression for the conductivity in extended states is given by equation (1.12). The occupancy of the electronic states in the conduction band (extended states) is given by the Fermi-Dirac distribution function, as presented in equation (1.12). The Fermi-Dirac distribution function decreases exponentially at high energy and will cause a very rapid decrease of the product $g(E) \cdot f(E)$, when the energy goes up from $\mathrm{E}_{\mathrm{C}}$. Therefore, one may treat the density of states and the mobility of carriers at the bottom of 
the conduction band as approximately constant $\left(\mathrm{N}_{\mathrm{C}}=10^{21} \mathrm{~cm}^{-3} \mathrm{eV}^{-1}\right.$ and $\mu_{\mathrm{C}}$, respectively). The conductivity in the extended states is then:

$$
\sigma_{0} \cong e \cdot \mu_{C} \cdot N_{C} \cdot \int_{E_{c}}^{\infty} f(E) d E
$$

For energy much greater than the Fermi level we have $E-E_{F 0} \gg k_{B} T$ and the Fermi-Dirac distribution function can be approximated as

$$
f(E)=\exp \left(-\frac{E-E_{F 0}}{k_{B} T}\right) \text {. }
$$

A relatively simple expression for the thermally activated conductivity in extended states is then obtained by substituting equation (3.2) into equation (3.1) and carrying out the integration.

$$
\sigma_{0} \cong e \cdot \mu_{C} \cdot N_{C} \cdot k_{B} T \cdot \exp \left(-\frac{E_{C}-E_{F 0}}{k_{B} T}\right)
$$

\subsection{Hopping Conductivity in the Localized States}

In the tail states, the main transport mechanism is hopping between the localized states. The expression for hopping conductivity in the conduction and valence bands tails $\left(E_{A}<E<E_{C}\right.$ or $E_{V}<E<E_{B}$, see figure 1.2), is given in equation (1.20). Therefore, the final expression for the hopping conductivity in the conduction band tail states is:

$$
\sigma_{\text {tail }}=e^{2} v_{p h} R^{2} N_{C} \exp \left(-\frac{E_{A}-E_{F n}+W_{1}}{k_{B} T}\right)
$$

where $E_{F n}$ is the quasi-Fermi level for the electrons.

The hopping conductivity due to holes was neglected. 


\subsection{Beta Conductivity}

The tritium decay process produces a $\beta$ particle, with an average energy of 5.69 $\mathrm{keV}$. Each $\beta$ particle will create over 1500 electron-hole pairs. These carriers are the contribution to the conductivity of the tritium decay process. The mechanism is similar to the photovoltaic effect, when the excess carriers are created by the interaction of photons with the amorphous network. The contribution to conductivity of the tritium decay process can be expressed as:

$$
\sigma_{\beta}=e \cdot \mu_{c} \cdot G_{\beta} \cdot \tau
$$

where $G_{\beta}$ is the generation rate of the electron-hole pairs, and $\tau$ is the carriers life-time. The transport takes place in the extended states and the carriers will have mobility $\mu_{C}$.

The carrier generation rate depends on the decay constant of tritium $(\lambda)$, atomic density of tritium in the amorphous silicon film $\left(n_{T}\right)$, average energy of a $\beta$ particle $\left(E_{\text {avg }}\right)$, the energy needed to produce an electron-hole pair by a $\beta$ particle $(\varepsilon)$, and the fraction of $\beta$ particles absorbed in the material (k). Equation (3.5) gives this dependence [9].

$$
G_{\beta}=\frac{k \lambda n_{T} E_{\text {avg }}}{\varepsilon}
$$

$$
\begin{array}{lll}
\mathrm{k} & = & 0.5[8] ; \\
\lambda & = & 1.78 \times 10^{-9} \mathrm{~s}^{-1} ; \\
\mathrm{E}_{\mathrm{avg}} & =5.69 \mathrm{keV} ; \\
\varepsilon & =3.4-4.4 \mathrm{eV} ; \\
\mathrm{n}_{\mathrm{T}} & =5-10 \% \text { of silicon atoms density. }
\end{array}
$$


The electron lifetime is greatly influenced by the presence of recombination centers that give rise to states in the energy band gap of the material. It depends also on the capture cross section of the recombination centers (C). Equation (3.6) gives the expression for the lifetime for recombination via recombination centers:

$$
\tau=\frac{1}{v_{T} \cdot C \cdot N_{R E C}}
$$

where $v_{T}=$ thermal velocity $\left(10^{7} \mathrm{~cm} / \mathrm{s}\right)$;

$C=$ recombination centers capture cross section $\left(10^{-16} \mathrm{~cm}^{2}\right)$;

$N_{R E C}=$ density of recombination centers.

The density of recombination centers $\left(N_{R E C}\right)$ is determined by the density of defect states in the gap, and by the probability that the defect states act as recombination centers. In this model, only the unoccupied dangling bonds ( $\mathrm{D}^{+}$centers) and the singly occupied dangling bonds ( $\mathrm{D}^{0}$ centers) are considered. These two types of dangling bonds are assumed to have Gaussian distributions (equations (3.7) and (3.8)), centered on $\mathrm{E}_{\mathrm{T}+}$ and $\mathrm{E}_{\mathrm{T} 0}$. Figure 3.1 shows the position of the dangling bonds in the band gap.

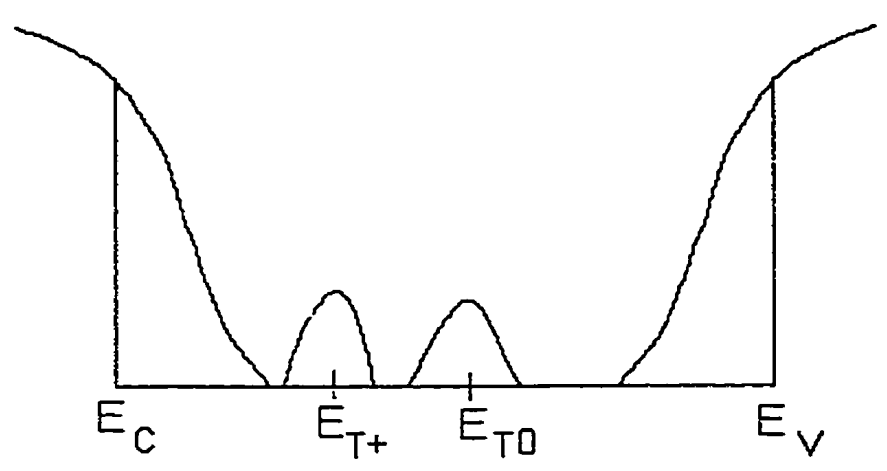

Figure 3.1. $D^{+}$and $D^{0}$ centers distribution in the band gap.

$$
N_{D+}(E)=\frac{N_{T_{+}}}{\sqrt{2 \pi s_{+}}} \exp \left(-\frac{\left(E-E_{T_{+}}\right)^{2}}{2 s_{+}^{2}}\right)
$$




$$
N_{D 0}(E)=\frac{N_{T 0}}{\sqrt{2 \pi} s_{0}} \exp \left(-\frac{\left(E-E_{T 0}\right)^{2}}{2 s_{0}^{2}}\right)
$$

where

$$
\begin{array}{ll}
N_{D+.0}(E) & =\text { density per unit energy of } \mathrm{D}^{+}\left(\mathrm{D}^{0}\right) \text { centers at energy } E \\
N_{T+.0} & =\text { density of } \mathrm{D}^{+}\left(\mathrm{D}^{0}\right) \text { centers; } \\
E_{T+.0} & =\text { peek position of the } \mathrm{D}^{+}\left(\mathrm{D}^{0}\right) \text { center distribution; } \\
S_{+, 0} & =\text { width of the } \mathrm{D}^{+}\left(\mathrm{D}^{0}\right) \text { center distribution. }
\end{array}
$$

The occupation probabilities cannot be determined simply from the Fermi-Dirac distribution function, because of the electronic correlation between the states.

The average number of electrons $n\left(E_{T}\right)=\frac{N}{N_{0}}$ in a defect site at energy $E_{T}$ is expressed as [12]:

$$
n\left(E_{T}\right)=2 f_{-}\left(E_{T}\right)+f_{0}\left(E_{T}\right)
$$

where

$$
\begin{array}{lll}
\mathrm{N} & = & \text { total number of electrons; } \\
\mathrm{N}_{0} & = & \text { total number of defect sites; } \\
f_{-, 0}\left(E_{T}\right) & = & \text { probability that the site is double (single) occupied. }
\end{array}
$$

The grand partition function for $N_{0}$ defects is [13]:

$$
Z=\left[1+2 \exp \left(-\frac{E_{T}-E_{F n}}{k_{B} T}\right)+\exp \left(-\frac{2 E_{T}-U-2 E_{F n}}{k_{B} T}\right)\right]^{N_{0}}
$$

where $U=$ correlation energy between defects.

The average number of electrons in a defect site at $E_{T}$ is [13]:

$$
n=\frac{1}{N_{0}} k_{B} T \frac{\partial \ln Z}{\partial E_{F n}}=\frac{2\left[\exp \left(-\frac{E_{T}-E_{F n}}{k_{B} T}\right)+\exp \left(-\frac{2 E_{T}-U-2 E_{F n}}{k_{B} T}\right)\right]}{1+2 \exp \left(-\frac{E_{T}-E_{F n}}{k_{B} T}\right)+\exp \left(-\frac{2 E_{T}-U-2 E_{F n}}{k_{B} T}\right)} .
$$


The occupation probabilities satisfy the relation:

$$
f_{-}\left(E_{T}\right)+f_{0}\left(E_{T}\right)+f_{+}\left(E_{T}\right)=1 .
$$

In order to determine the occupation functions, we need a third relation. The average total energy $E\left(E_{T}\right)$ is [12]:

$$
E\left(E_{T}\right)=\left(2 E_{T}+U\right) \cdot f_{-}\left(E_{T}\right)+E_{T} \cdot f_{0}\left(E_{T}\right)
$$

By using the same grand partition function, one can determine another expression for the total energy at $E_{\mathrm{T}}$. This expression and equations (3.9), (3.11), and (3.12), can be used to calculate the occupation functions for defect states: $f_{+}(E)$ (for $D^{+}$centers) and $f_{0}(E)$ (for $D^{0}$ centers).

$$
\begin{aligned}
& f_{+}(E)=\frac{1}{1+2 \exp \left(-\frac{E-E_{F n}}{k_{B} T}\right)+\exp \left(-\frac{2 E-2 E_{F n}+U}{k_{B} T}\right)}, \\
& f_{0}(E)=\frac{2 \exp \left(-\frac{E-E_{F n}}{k_{B} T}\right)}{1+2 \exp \left(-\frac{E-E_{F n}}{k_{B} T}\right)+\exp \left(-\frac{2 E-2 E_{F n}+U}{k_{B} T}\right)} .
\end{aligned}
$$

The total number of recombination centers is the integral over the band gap of the active recombination centers (equation 3.16).

$$
N_{R E C}=\int_{E_{v}}^{E_{C}}\left(N_{D+}(E) \cdot f_{+}(E)+N_{D 0}(E) \cdot f_{0}(E)\right) d E .
$$




\subsection{Modeling the Electrical Conductivity}

The model used in the numerical simulation considers only thermally activated conductivity $\left(\sigma_{0}\right)$, hopping conductivity in the localized states $\left(\sigma_{\text {tail }}\right)$ and beta conductivity $\left(\sigma_{\beta}\right)$. Equation (3.17) represents the global relation used to model the conductivity.

$$
\sigma=\sigma_{0}+\sigma_{\text {tail }}+\sigma_{\beta}
$$

\subsection{Initial Experimental Results}

The main purpose of the numerical simulation was to help develop an understanding of the transport mechanisms due to the tritium decay. Therefore, the simulation results have to be compared with experimental data.

Hydrogenated-tritiated amorphous silicon films were deposited by using the dc saddle-field deposition system, presented in chapter 2 . The samples were prepared by Mr. Tome Kosteski at the Ontario Hydro Tritium Laboratory.

The deposition conditions are presented in table 1 . The gas pressure, the substrate and anode bias and the flows of the gases are adjustable parameters and the anode and substrate currents are measured for a rough evaluation of the process.

Table 1. Deposition conditions

\begin{tabular}{|c|c|c|c|c|c|c|c|c|}
\hline Parameter & $\begin{array}{c}\text { Gas } \\
\text { pressure }\end{array}$ & $\begin{array}{c}\text { Anode } \\
\text { bias }\end{array}$ & $\begin{array}{c}\text { Anode } \\
\text { current }\end{array}$ & $\begin{array}{c}\text { Substrate } \\
\text { bias }\end{array}$ & $\begin{array}{c}\text { Substrate } \\
\text { current }\end{array}$ & $\begin{array}{c}\mathrm{T}_{2} \\
\text { flow }\end{array}$ & $\begin{array}{c}\mathrm{SiH}_{4} \\
\text { flow }\end{array}$ & $\begin{array}{c}\text { Deposition } \\
\text { Time }\end{array}$ \\
\hline Value & $90 \mathrm{mTorr}$ & $550 \mathrm{~V}$ & $15 \mathrm{~mA}$ & $0 \mathrm{~V}$ & $2 \mathrm{~mA}$ & $3 \mathrm{sccm}$ & $5 \mathrm{sccm}$ & 1.5 hours \\
\hline
\end{tabular}

Corning 7059 Glass substrates were used for the samples designated for electrical conductivity measurements. Two coplanar pads of $\mathrm{Ni}-\mathrm{Cr}$ with a gap of $0.1 \mathrm{~cm}$ were evaporated onto the film in order to provide ohmic contacts. Teflon insulated wires were connected to each pad using silver paste. A bias of $10 \mathrm{~V}$ was applied between the contacts 
and the current was measured with an electrometer, while the temperature was changed in steps of $5^{\circ} \mathrm{C}$. The measurement procedure was repeated as a function of time.

Figure 3.2 presents the experimental data for the conductivity versus temperature, as a function of time. One can see that the low temperature conductivity is determined by $\beta$ - conductivity. At high temperature an exponential increase in the conductivity is observed. This exponential increase is associated with thermally activated conductivity in the extended states.

Figure 3.3 shows the evolution of conductivity with time, at $185^{\circ} \mathrm{C}$. During the first week after deposition, the conductivity decreases with time.

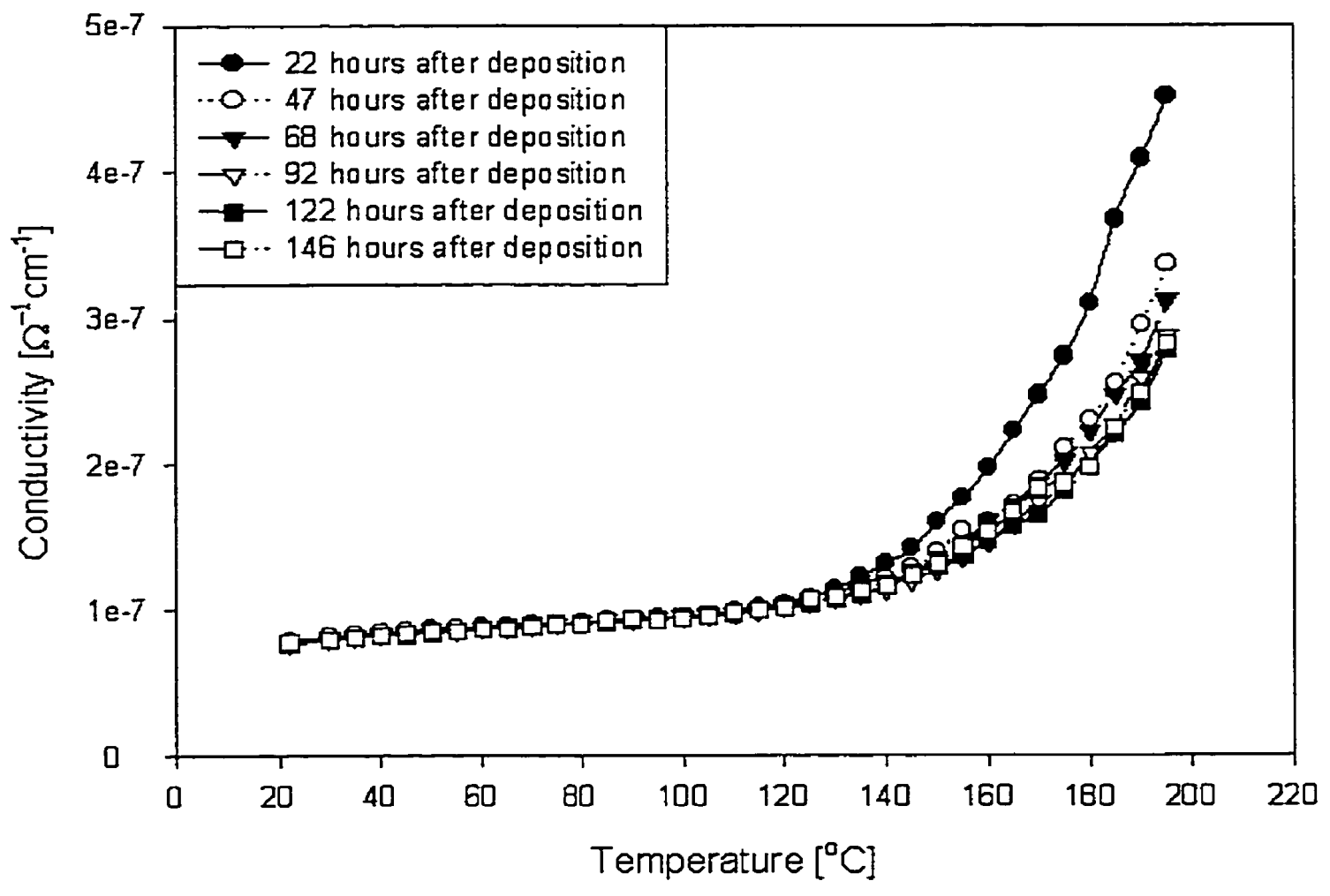

Figure 3.2. Initial experimental results. 


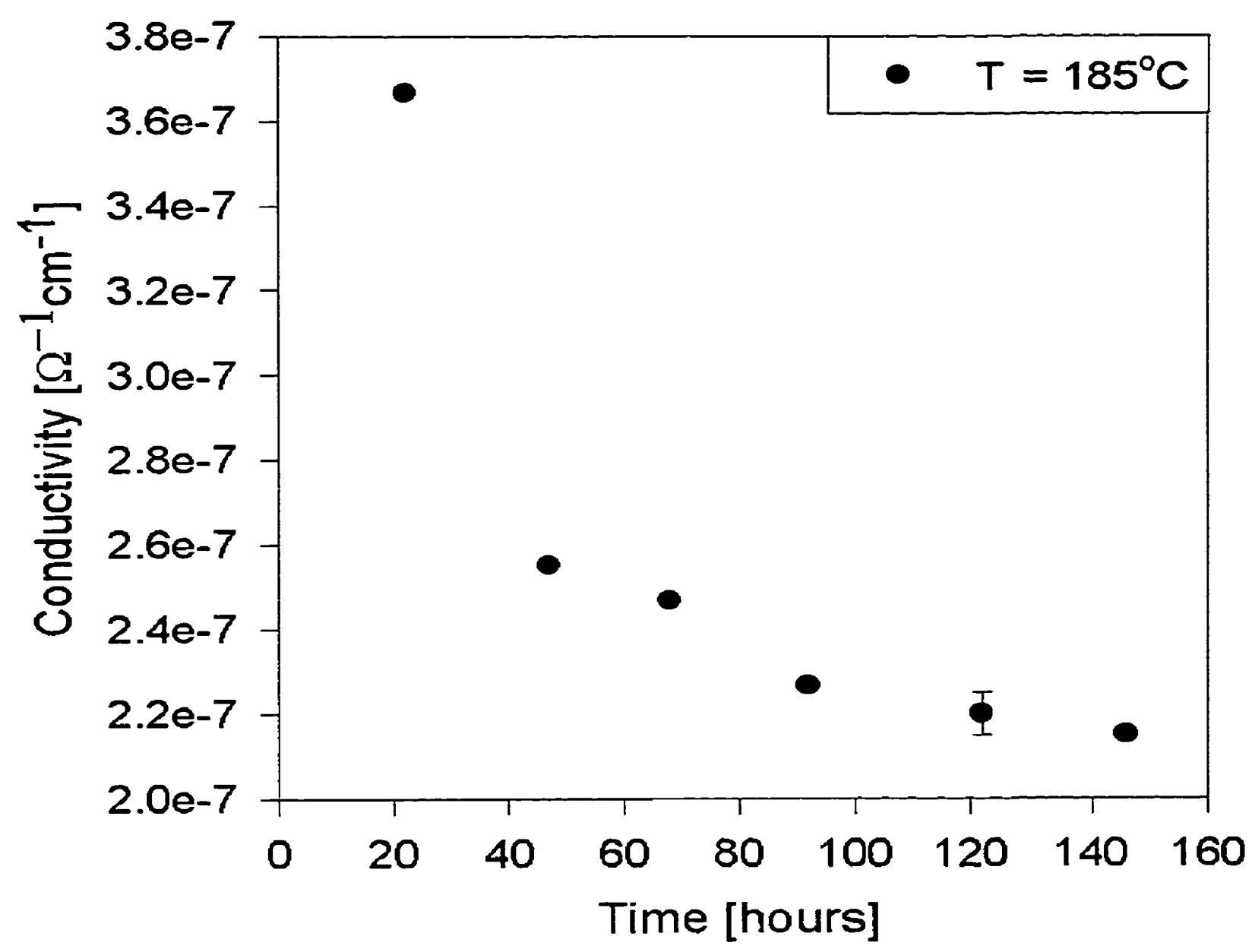

Figure 3.3 Initial experimental results.

The experimental data used in this thesis were obtained by Mr. Tome Kosteski, and Dr. Franco Gaspari. 


\section{Chapter IV}

\section{Results}

The model described by equation (3.17) was used to fit the experimental data presented in figures 3.2 and 3.3.

\subsection{Fermi Energy}

As was mentioned before, at high temperature thermally activated conductivity dominates. Considering this, equation (3.18) can be simplified to

$$
\sigma \cong \sigma_{0} \equiv e \cdot \mu_{c} \cdot N_{C} \cdot k_{B} T \cdot \exp \left(-\frac{E_{C}-E_{F 0}}{k_{B} T}\right) .
$$

By fitting equation (4.1), to the experimental data, the Fermi level was determined. In figure 4.1 is presented the time evolution of the Fermi energy. These values were subsequently used in the fitting procedure.

\subsection{Fitting Procedure}

Random values were generated for the dangling bonds concentrations $\left(N_{D+}\right.$ and $N_{D 0}$ ), quasi Fermi level $\left(E_{F n}\right)$ and for the hopping radius $(R)$. Using these random values in equations (3.3) - (3.9), (3.17) and (3.18), the theoretical values for the electrical conductivity were obtained. These were compared with the experimental data and the set that minimized the difference, the minimum fitting error, was determined. The expression used for evaluating the fitting error is presented in equation (4.2). 


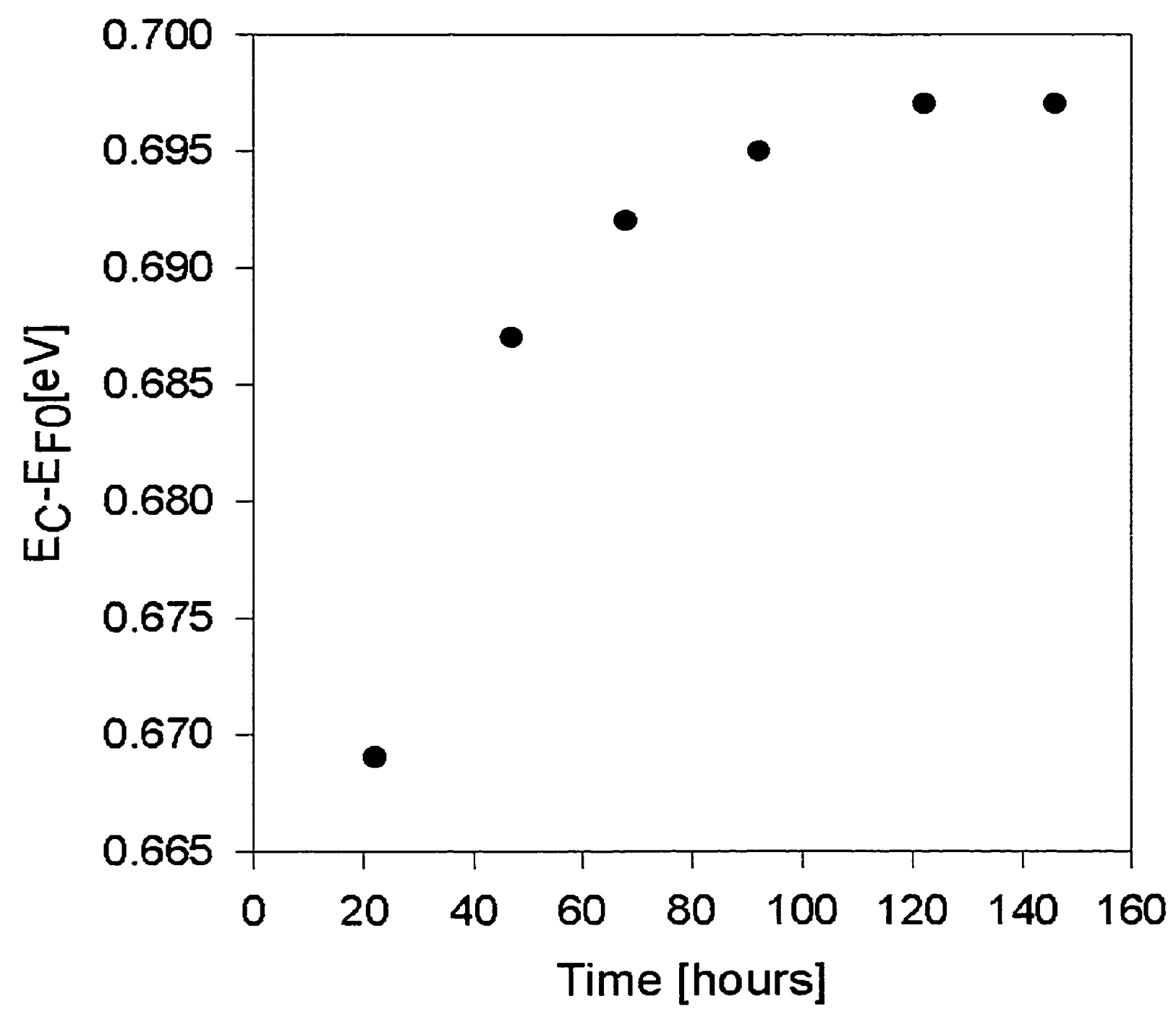

Figure 4.1. Time evolution of the Fermi energy

$$
\text { error }=\max \left[\operatorname{abs}\left(\frac{\sigma_{\text {exp }}-\sigma_{\text {calculated }}}{\sigma_{\text {exp }}}\right)\right] \cdot 100 \%
$$

where

$$
\begin{array}{lll}
\sigma_{\text {exp }} & =\text { experimental values for the conductivity; } \\
\sigma_{\text {calculated }} & =\quad \text { calculated values for the conductivity. }
\end{array}
$$

Table 4.1 gives the literature values of parameters used in the fitting. The referenced values were slightly adjusted in order to optimize the fit. This way the fitting 
error was reduced from $15 \%$ to around $6 \%$. The adjusted values are also presented in table 4.1. Figures 4.2 to 4.7 present the fitted curves for the conductivity measured after different periods of time (22 hours to 146 hours).

Table 4.1 Parameters used in the model

\begin{tabular}{|c|c|c|c|}
\hline Parameter & $\begin{array}{c}\text { Used value } \\
\text { (adjusted) }\end{array}$ & Literature value & Reference \\
\hline $\begin{array}{c}\text { Capture cross section for dangling } \\
\text { bonds }(\mathrm{C})\end{array}$ & $10^{-16} \mathrm{~cm}^{2}$ & $10^{-16} \mathrm{~cm}^{2}$ & {$[14]$} \\
\hline Peak position for $\mathrm{D}^{+}$centers $\left(\mathrm{E}_{\mathrm{T}+}\right)$ & $0.35 \mathrm{eV}$ from $\mathrm{E}_{\mathrm{C}}$ & $0.37 \mathrm{eV}$ from $\mathrm{E}_{\mathrm{C}}$ & {$[15]$} \\
\hline Peak position for $\mathrm{D}^{0}$ centers $\left(\mathrm{E}_{\mathrm{T} 0}\right)$ & $0.66 \mathrm{eV}$ from $\mathrm{E}_{\mathrm{C}}$ & $0.68 \mathrm{eV}$ from $\mathrm{E}_{\mathrm{C}}$ & {$[15]$} \\
\hline Correlation energy $(\mathrm{U})$ & $0.3 \mathrm{leV}$ & $0.31 \mathrm{eV}$ & {$[15]$} \\
\hline Localized states range near $\mathrm{E}_{\mathrm{C}}\left(\mathrm{E}_{\mathrm{A}}\right)$ & $0.4 \mathrm{eV}$ & $0.3 \mathrm{eV}$ & {$[6]$} \\
\hline Hopping energy $(\mathrm{W})$ & $0.19 \mathrm{eV}$ & $0.19 \mathrm{eV}$ & {$[6]$} \\
\hline $\begin{array}{c}\text { Dangling bonds distribution widths } \\
\left(\mathrm{s}_{+} \text {and } \mathrm{s}_{0}\right)\end{array}$ & $0.07 \mathrm{and} 0.1 \mathrm{l}$ & 0.1 & {$[11]$} \\
\hline
\end{tabular}




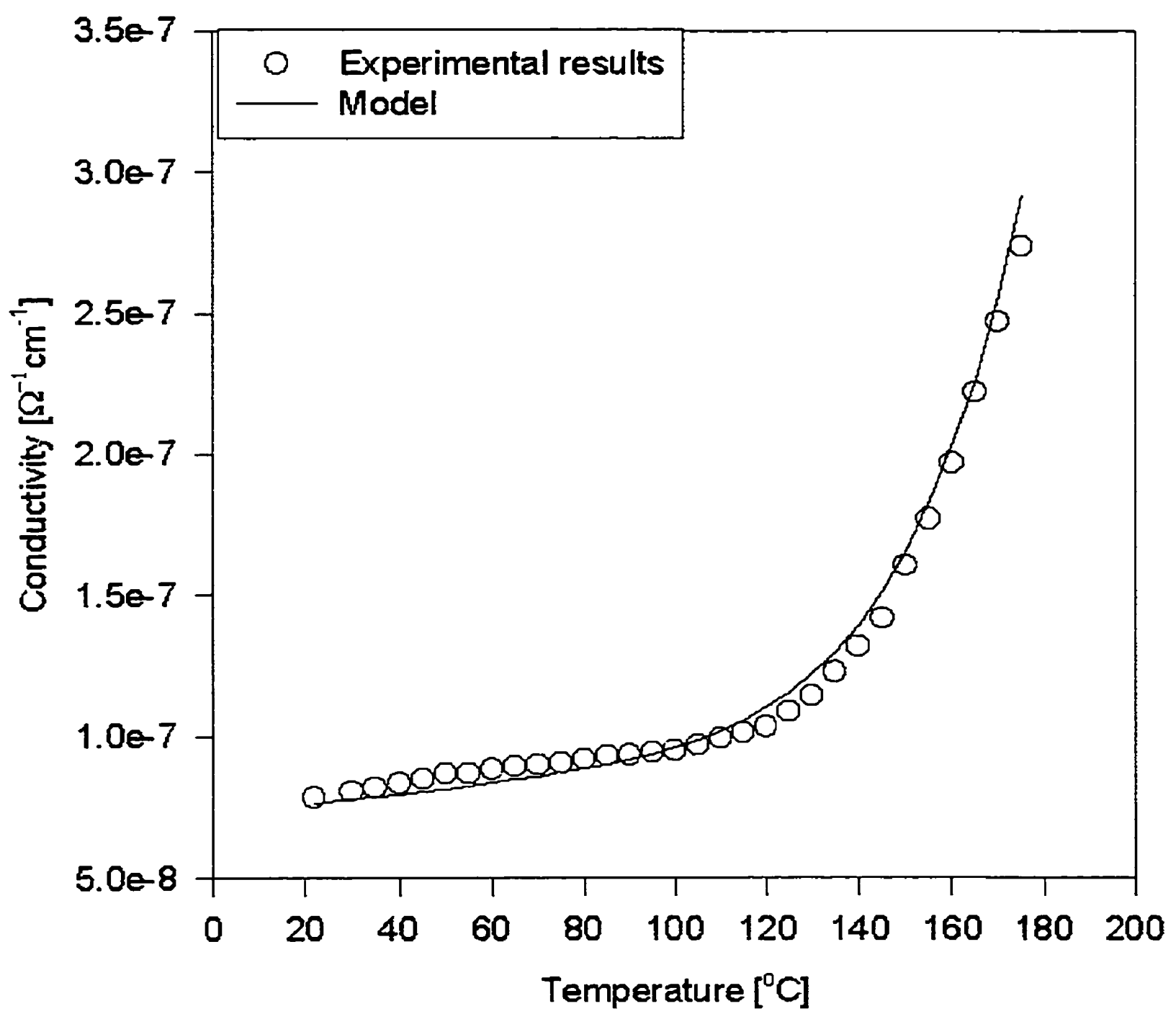

Figure 4.2. Experimental results and the fitted curve for conductivity,

22 hours after deposition (6\% error) 


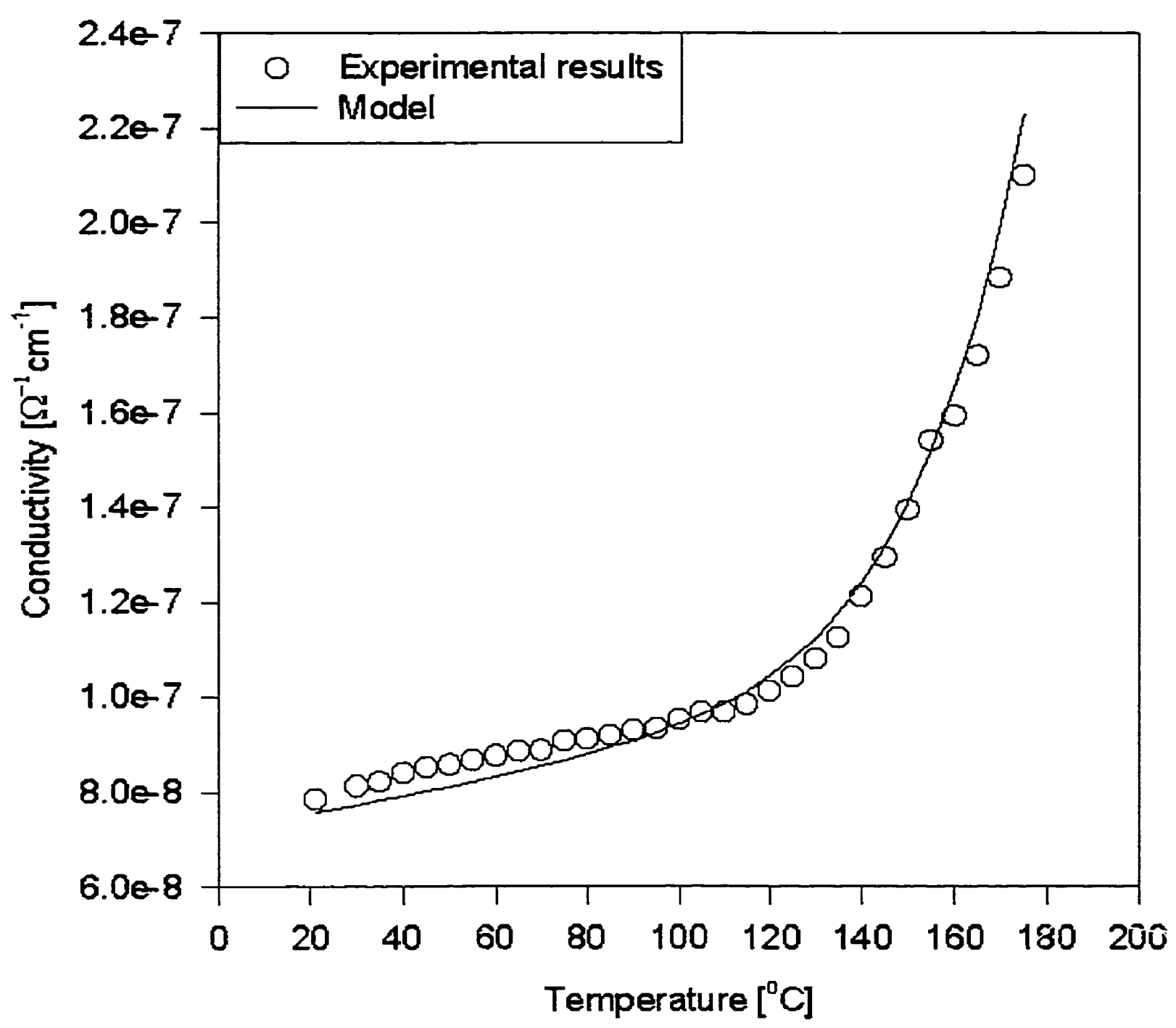

Figure 4.3. Experimental results and the fitted curve for conductivity,

47 hours after deposition ( $6 \%$ error) 


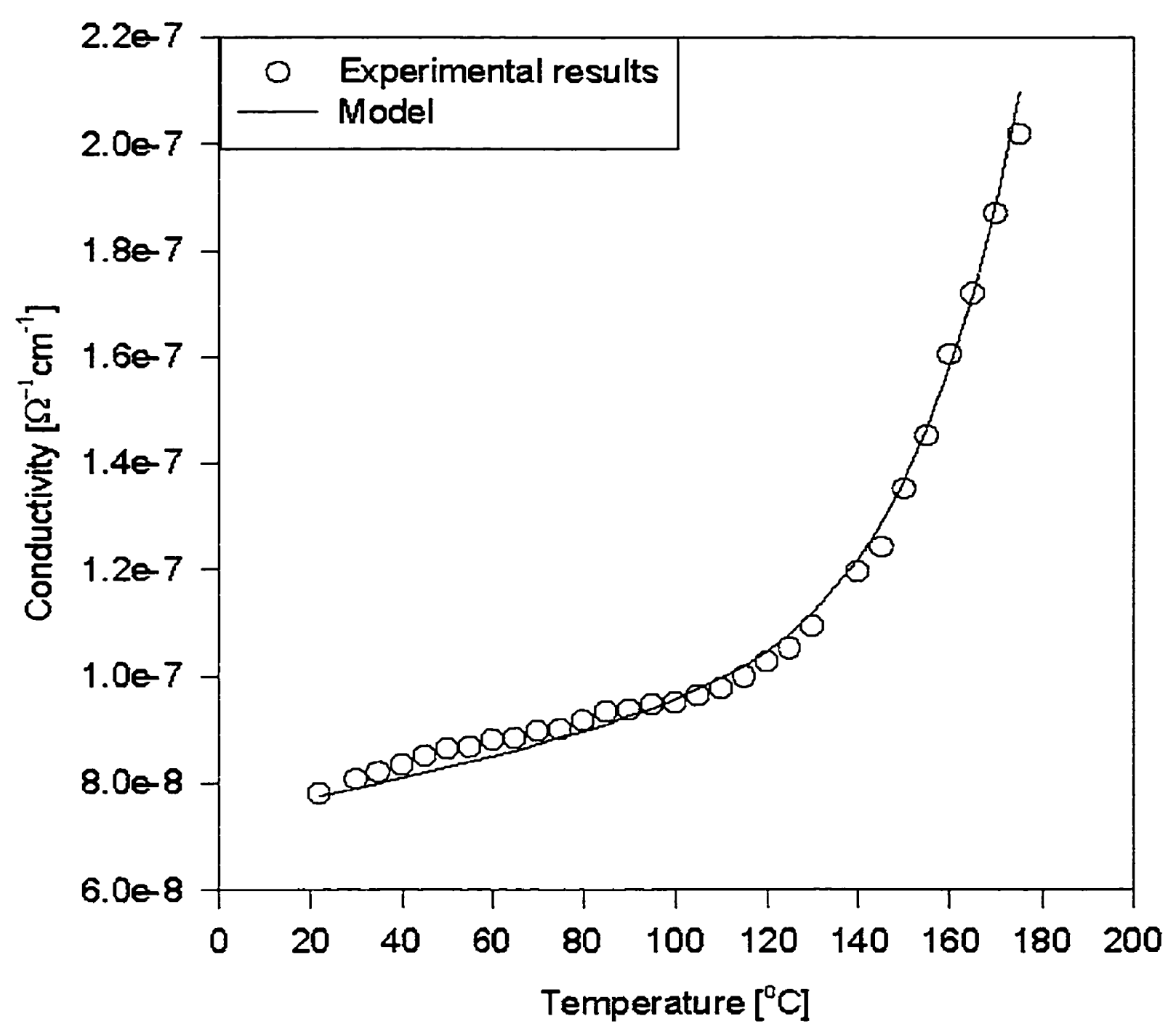

Figure 4.4. Experimental results and the fitted curve for conductivity, 68 hours after deposition ( $4 \%$ error) 


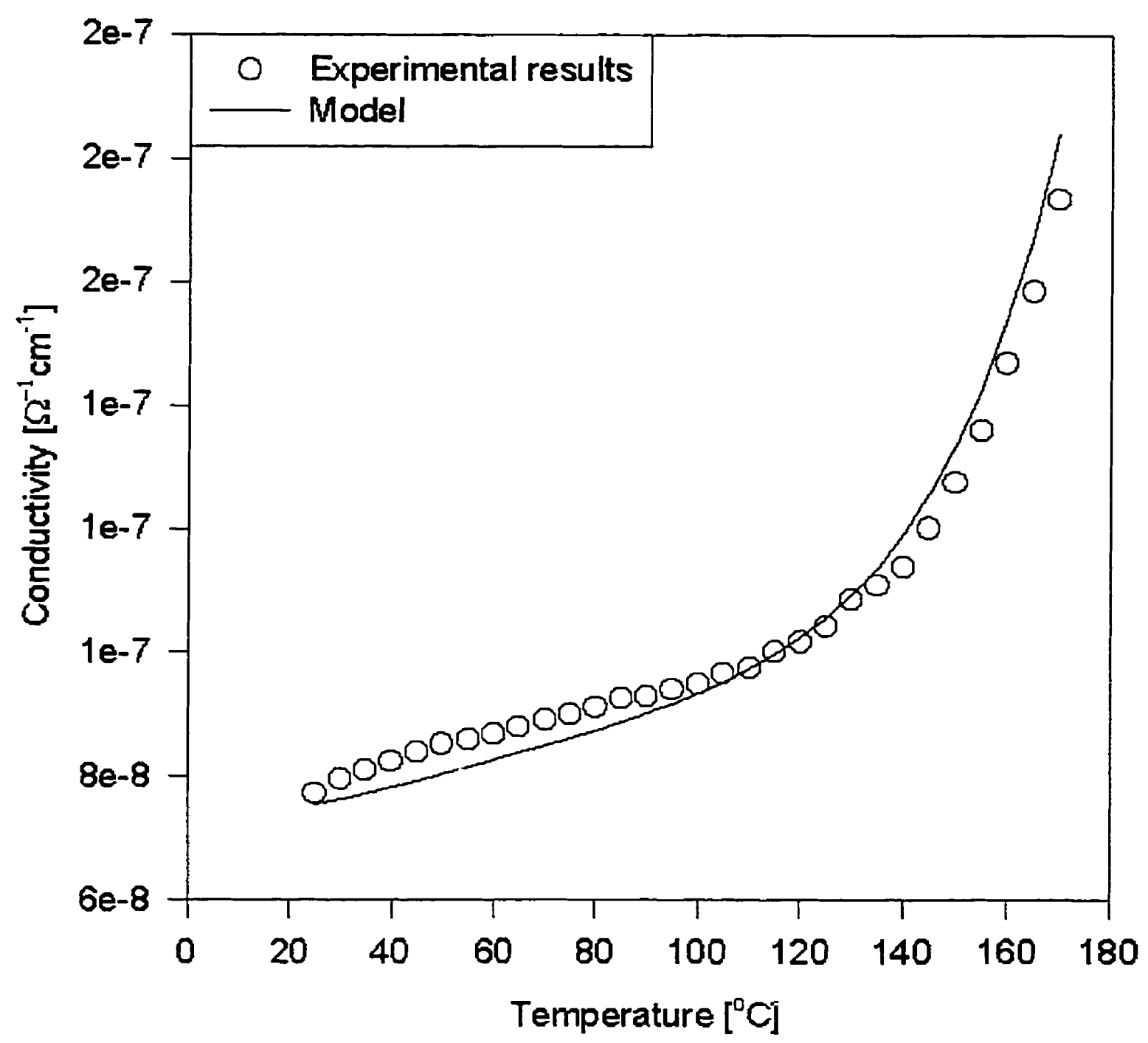

Figure 4.5. Experimental results and the fitted curve for conductivity,

92 hours after deposition ( $5 \%$ error) 


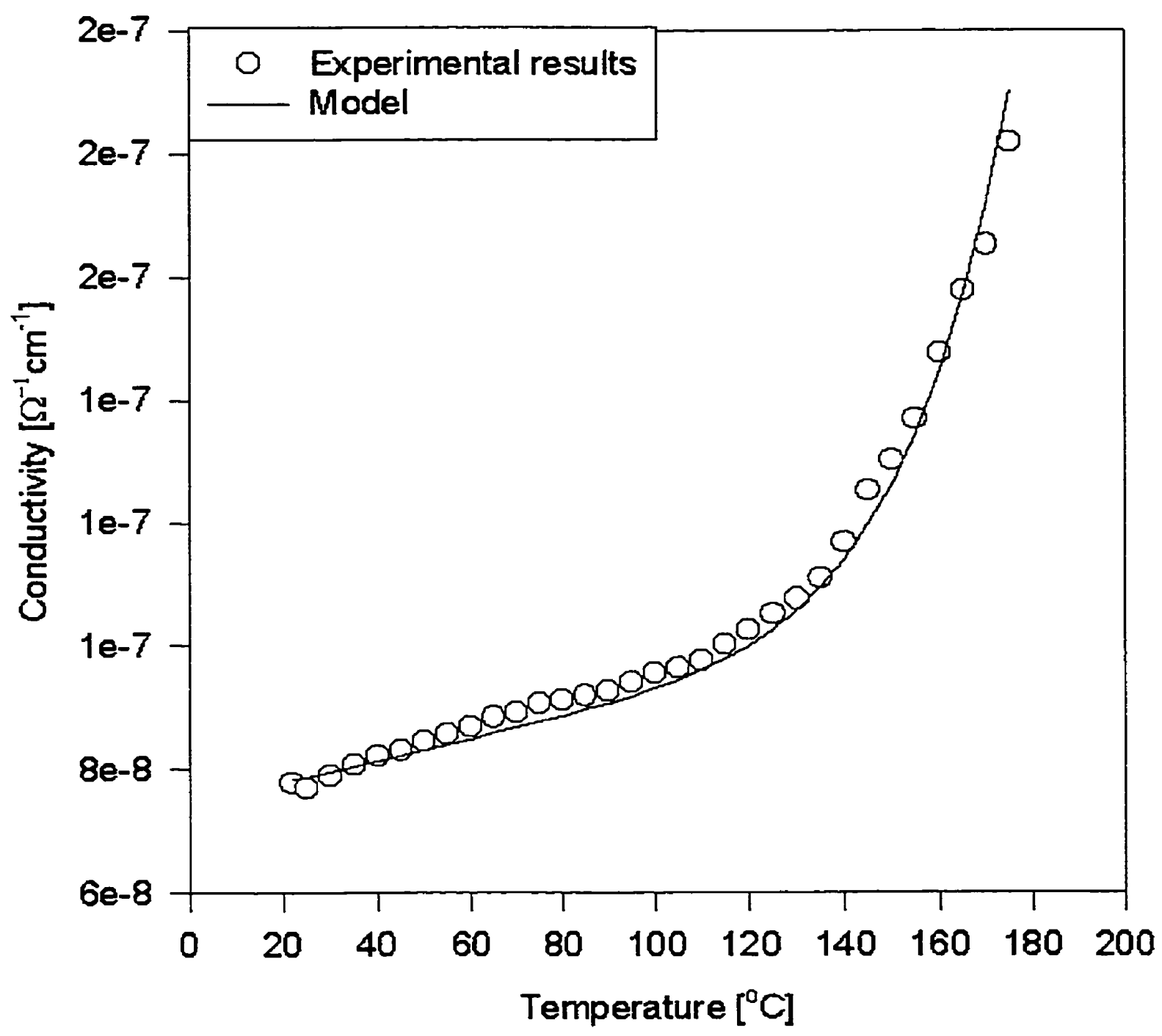

Figure 4.6. Experimental results and the fitted curve for conductivity, 122 hours after deposition (4\% error) 


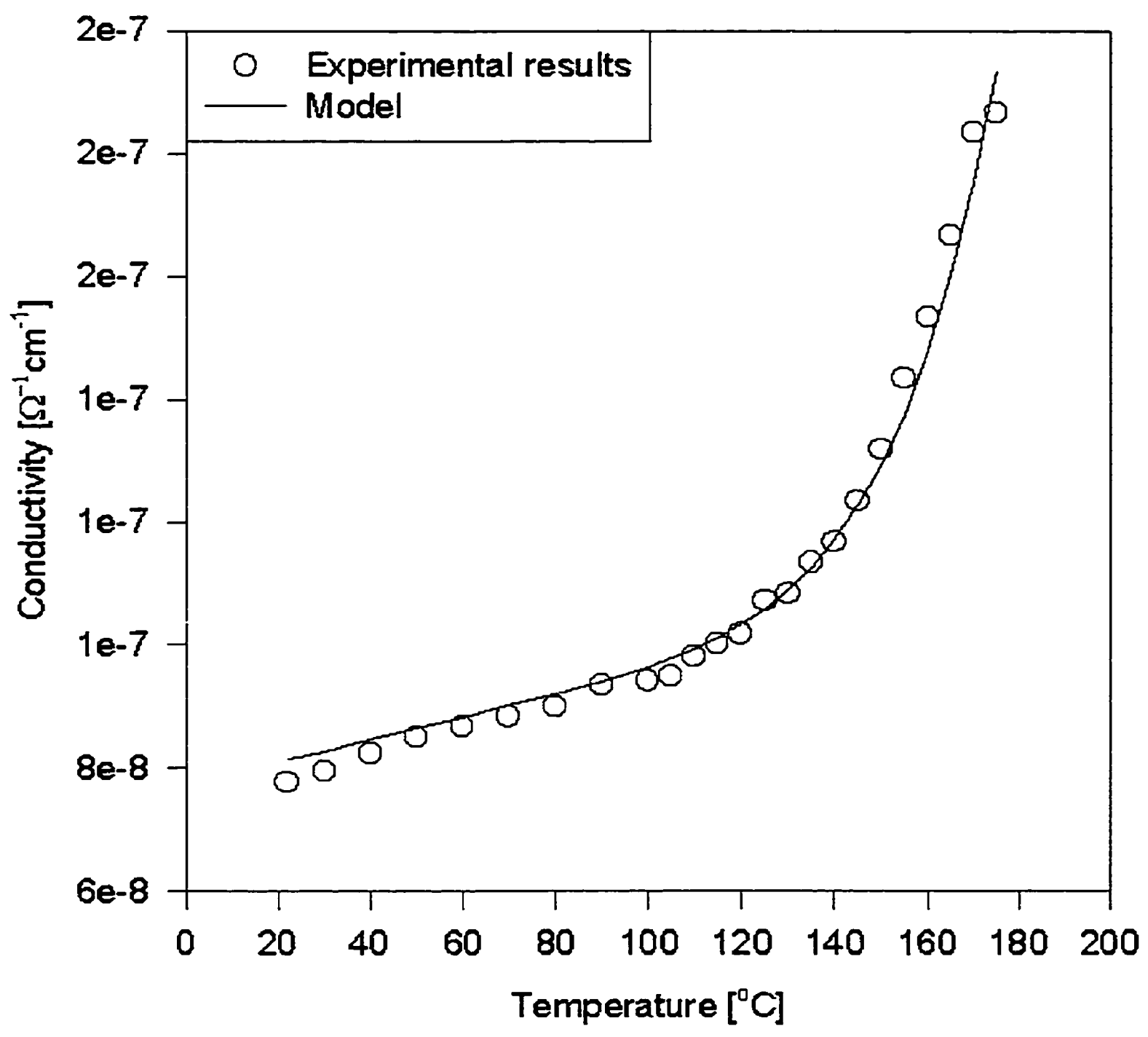

Figure 4.7. Experimental results and the fitted curve for conductivity, 146 hours after deposition ( $4 \%$ error) 


\subsection{Results}

The results of the fitting process are presented in figures 4.8 to 4.11 .

Figure 4.8 shows the time evolution of the quasi - Fermi energy for electrons. This parameter affects both the beta conductivity in extended states and the hopping conductivity in localized states.

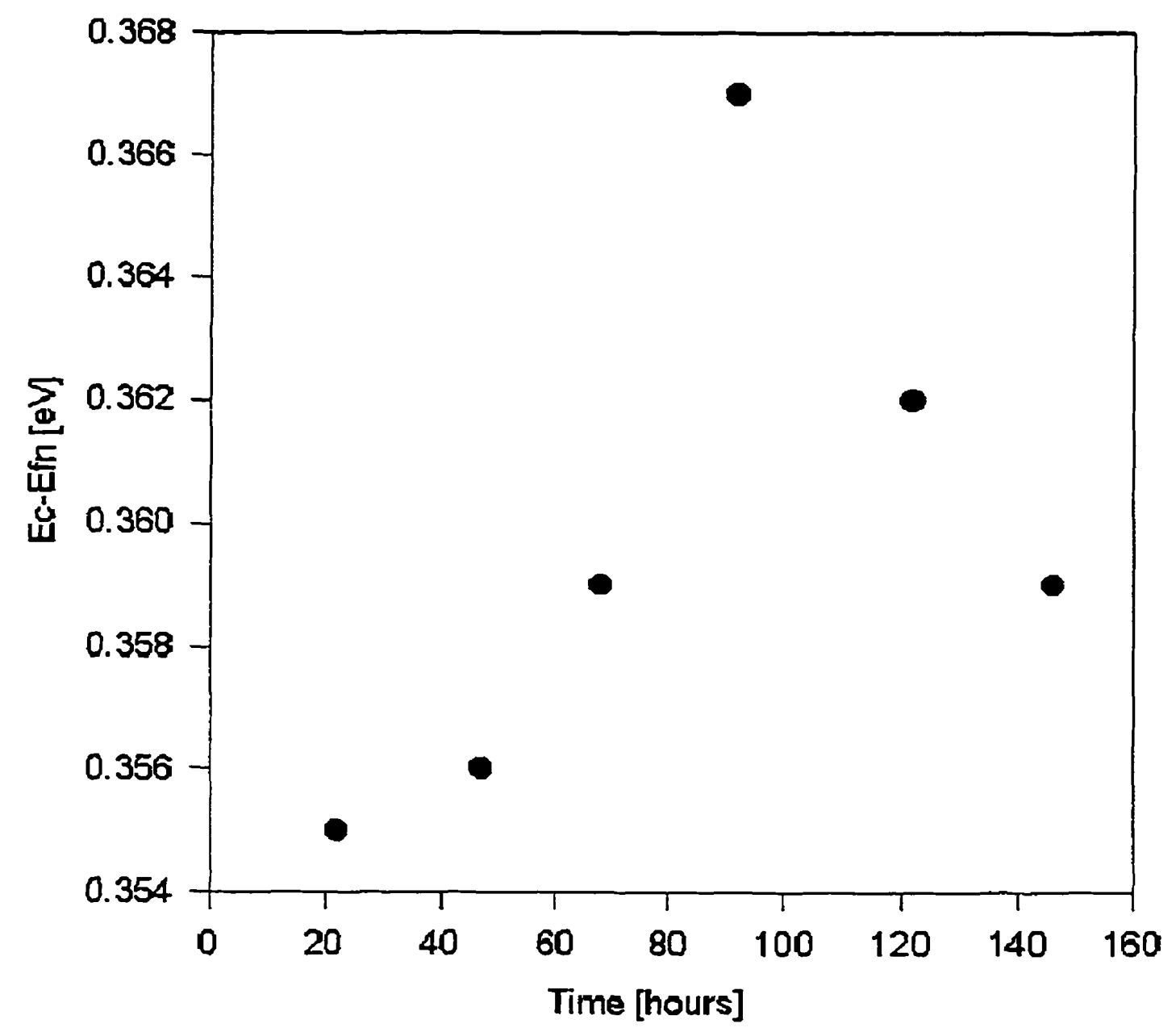

Figure 4.8. Time evolution of the quasi - Fermi energy for electrons 
Figure 4.9 presents the values for the concentration of the neutrally charged dangling bonds and positively charged dangling bonds. They could be determined separately because they have different probabilities to act as recombination centers.

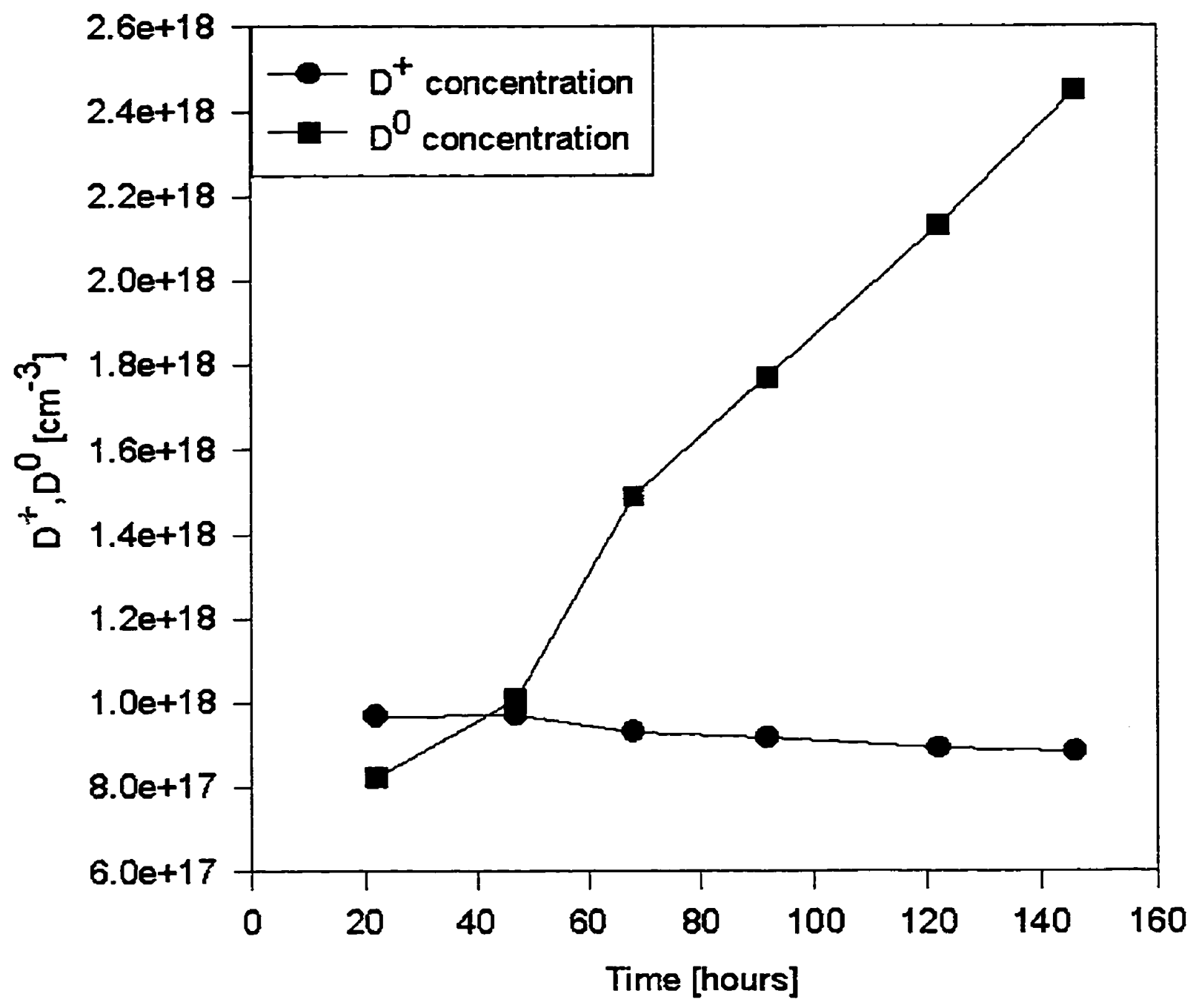

Figure 4.9. Time evolution of the dangling bonds concentrations 
The total defect concentration is presented in figure 4.10.

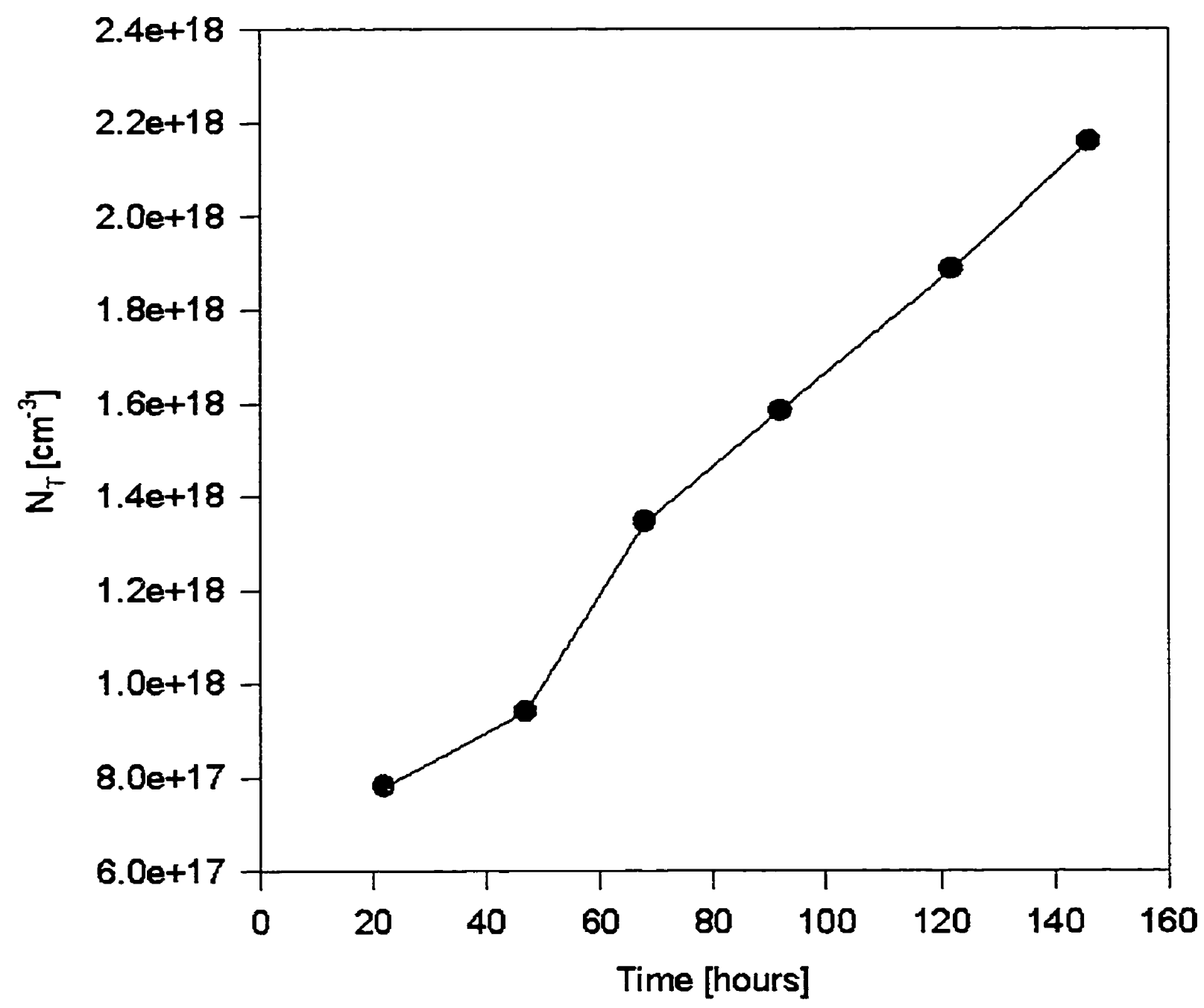

Figure 4.10. Time evolution of the total dangling bonds concentration 
The results for the hopping distance are presented in figure 4.11. This parameter will affect the hopping conductivity in the localized states.

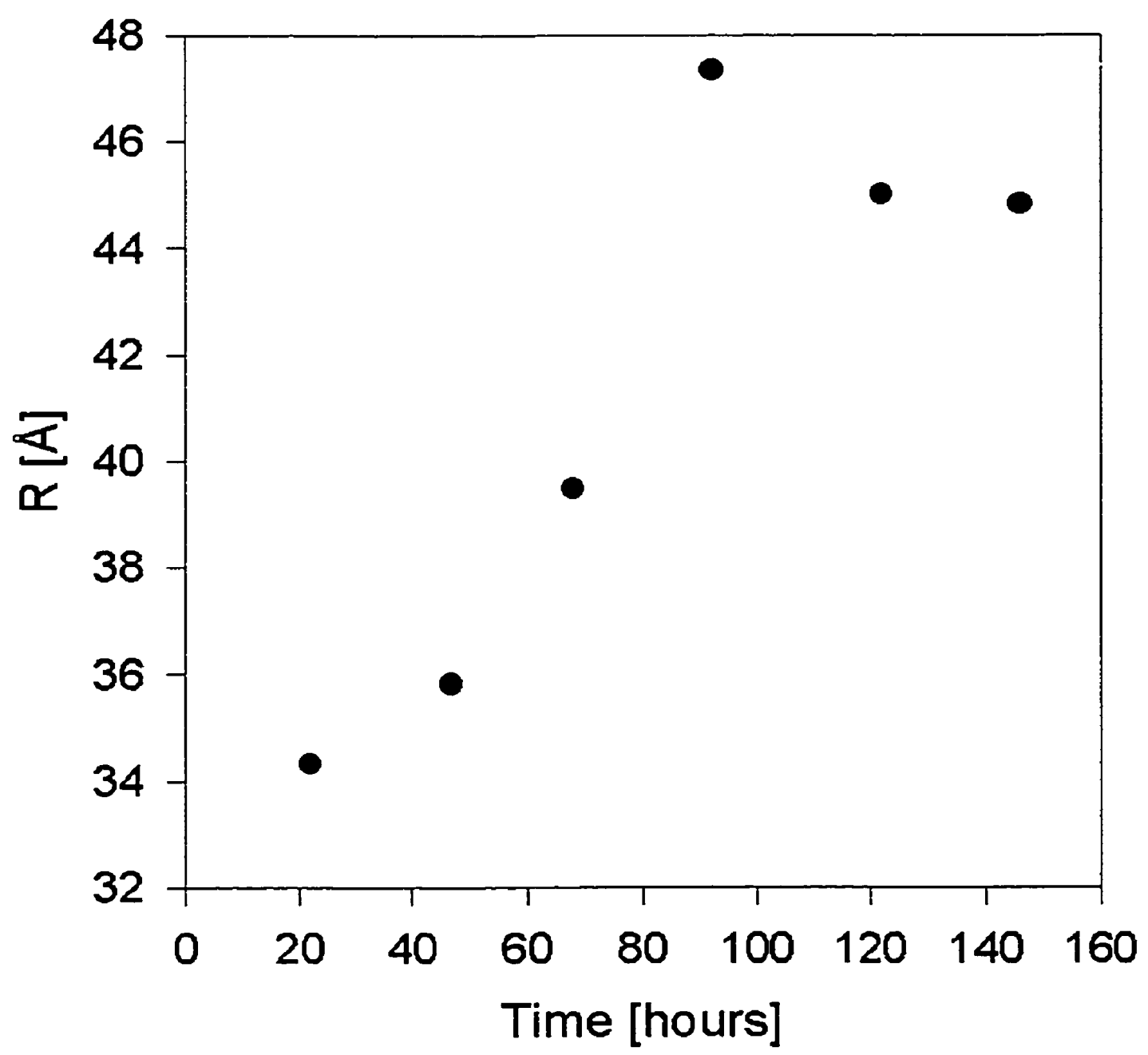

Figure 4.11. Time evolution of the hopping distance 


\section{Chapter V}

\section{Discussion}

The purpose of this study was to explain the change in electrical conductivity with time for tritiated amorphous silicon samples. Therefore, the most important result is the one that can justify the decrease in conductivity.

In figure 4.10 it can be seen that the total dangling bond concentration increases with time. The dangling bonds act as recombination centers. The increase in the recombination center concentration decreases the carrier lifetime. This in turn decreases excess carrier concentration and therefore decrease the electrical conductivity related to the beta decay.

In figure 4.9 are presented the time evolution of the positively charged and neutral dangling bond concentrations. One can see that the concentration of the neutral dangling bonds increases significantly in time, while the concentration of the positively charged dangling bonds decreases slightly. This behavior can be explained by the mechanism shown schematically in figure 5.1.

As one can see in equation (2.1), tritium decays into a positively charged helium ion and an energetic electron ( $\beta$ particle). The $\beta$ particle will collide with the lattice, forming over 1500 electron - hole pairs. This electron-hole pair creation process is similar to photo-conductivity and justifies the use of equation (3.5) to describe beta-conductivity.

The helium ion has a very high probability of attracting an electron and becoming a neutral helium atom. The neutral helium atom will likely diffuse away. This process does not have an important effect on the surrounding lattice. 


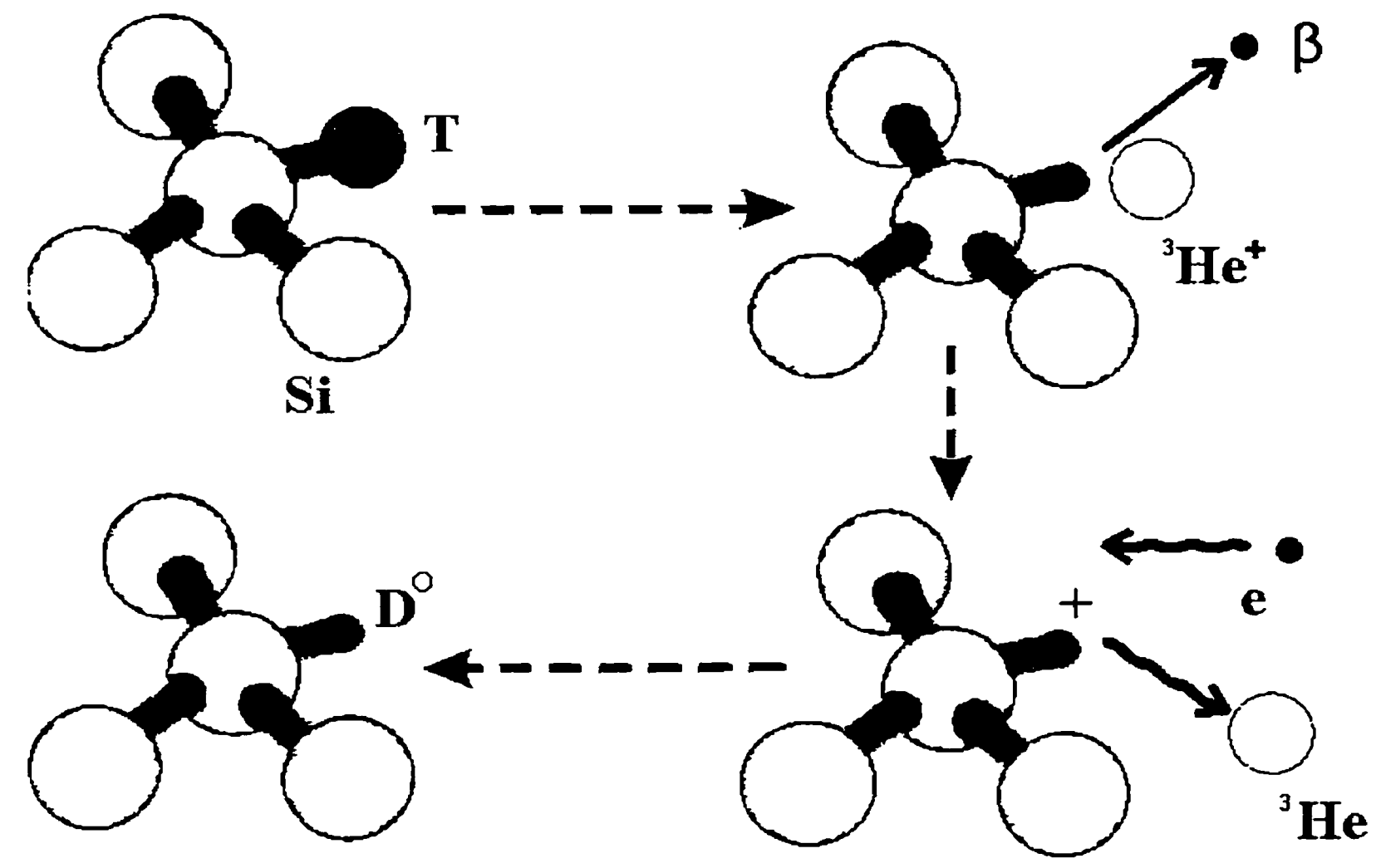

Figure 5.1. The mechanism of tritium decay and dangling bond formation.

The site that was initially occupied by the tritium atom, bonded with silicon, is left empty. The helium ion has attracted both electrons involved in the covalent bond between tritium and silicon. Therefore, the unsatisfied bond of the silicon atom is a positively charged dangling bond, a $\mathrm{D}^{+}$center. Due to the $\beta$ particle collisions, there are many free electrons created in the material. The $\mathrm{D}^{+}$centers will rapidly attract electrons, forming neutral dangling bonds, $\mathrm{D}^{0}$ centers. In time, more tritium atoms decay and more $\mathrm{D}^{0}$ centers are created. This explains the increase in the neutral dangling bond concentration. 
Initially, a competition between the $\mathrm{D}^{+}$center creation and the $\mathrm{D}^{0}$ center creation will likely take place. The $\mathrm{D}^{0}$ centers creation will dominate eventually, due to the large number of free electrons available to be attracted by the $\mathrm{D}^{+}$centers.

Crandall suggested that in hydrogenated amorphous silicon there is a dynamic process of $\mathrm{D}^{+}$to $\mathrm{D}^{0}$ conversion $[10]$. The $\mathrm{D}^{+}$state is close to the bottom of the conduction band. When it attracts an electron it can be readily thermally ionized back to the original charged state. However, a fraction of these states will stabilize as $D^{0}$ states. Lattice relaxation is associated with the stabilization. The $\mathrm{D}^{0}$ state is deeper in the energy gap than the $\mathrm{D}^{+}$state, and a $15^{\circ}$ change in the bond angle is necessary for stabilization. In tritiated amorphous silicon the energy needed for this relaxation is derived from the nuclear decay process: the average energy of the $\beta$ particle is $5.6 \mathrm{keV}$ and the helium ion recoil energy is $3 \mathrm{eV}$. This explains the slight decrease with time in the $\mathrm{D}^{+}$content.

The decay constant of tritium is $\lambda=1.78 \cdot 10^{-9} \mathrm{~s}^{-1}[8]$. Therefore, the time evolution of the atomic concentration of tritium in tritiated amorphous silicon can be expressed by equation (5.1)

$$
N_{T}(t)=N_{\pi} \cdot \exp (-\lambda t)
$$

where $N_{T}(t)=\quad$ atomic concentration of tritium at the moment $t ;$

$\mathrm{N}_{\mathrm{TI}}=$ initial atomic concentration of tritium, just after deposition;

As it was shown before, each decaying tritium atom will create a dangling bond. The concentration of the dangling bonds created by the tritium decay will then be equal with the concentration of the decayed tritium atoms (equation (5.2)).

$$
N_{D}(t)=N_{T I}-N_{T}(t)
$$

where $N_{D}(t)=$ concentration of the dangling bonds created by the tritium decay. 
The total concentration of the dangling bonds calculated by using the model includes also the initial concentration of dangling bonds present previously in the material due to the deposition process. Considering the initial dangling bonds and equations (5.1) and (5.2), the total concentration of dangling bonds at time $t$ after the deposition can be predicted by using equation (5.3).

$$
N_{D}(t)=N_{D I}+N_{\pi}(1-\exp (-\lambda t))
$$

where $\mathrm{N}_{\mathrm{DI}}=$ initial concentration of dangling bonds.

The values of the dangling bonds concentration calculated using the model are shown in figure 5.2, along with the fitted curve, determined using equation (5.3). The fitting error was under $6.5 \%$.

From this fitting process we get the value of the initial concentration of dangling bonds of $\mathrm{N}_{\mathrm{DI}}=1.62 \cdot 10^{18} \mathrm{~cm}^{-3}$, and an atomic concentration of tritium of $\mathrm{N}_{\pi}=1.85 \cdot 10^{21} \mathrm{~cm}^{-3}$, which represents $3.5 \%$ of the atomic density of silicon $\left(5 \cdot 10^{22} \mathrm{~cm}^{-3}\right)$. The atomic concentration of tritium for this sample was determined using IR spectroscopy to be between $3 \%$ and $4 \%$ of the atomic concentration of silicon. The calculated value is in good agreement with this measurement. As one can see, the model can be used to calculate the initial concentration of tritium.

The results for quasi-Fermi energy for electrons are in agreement with the literature. In [14], a range for the quasi-Fermi level of between $0.1 \mathrm{eV}$ and $0.78 \mathrm{eV}$ from the bottom of the conduction band is suggested. As one can see in figure 4.8 , the calculated values are within this range.

The time evolution of the Fermi energy can be explained by the evolution of neutral dangling bond concentration. The concentration of the neutral dangling bonds 
increases with time. The states associated with these defects are deep into the gap. Therefore, the increase in the density of gap states will cause a shift of the Fermi energy towards the midgap, as one can see in figure 4.1 .

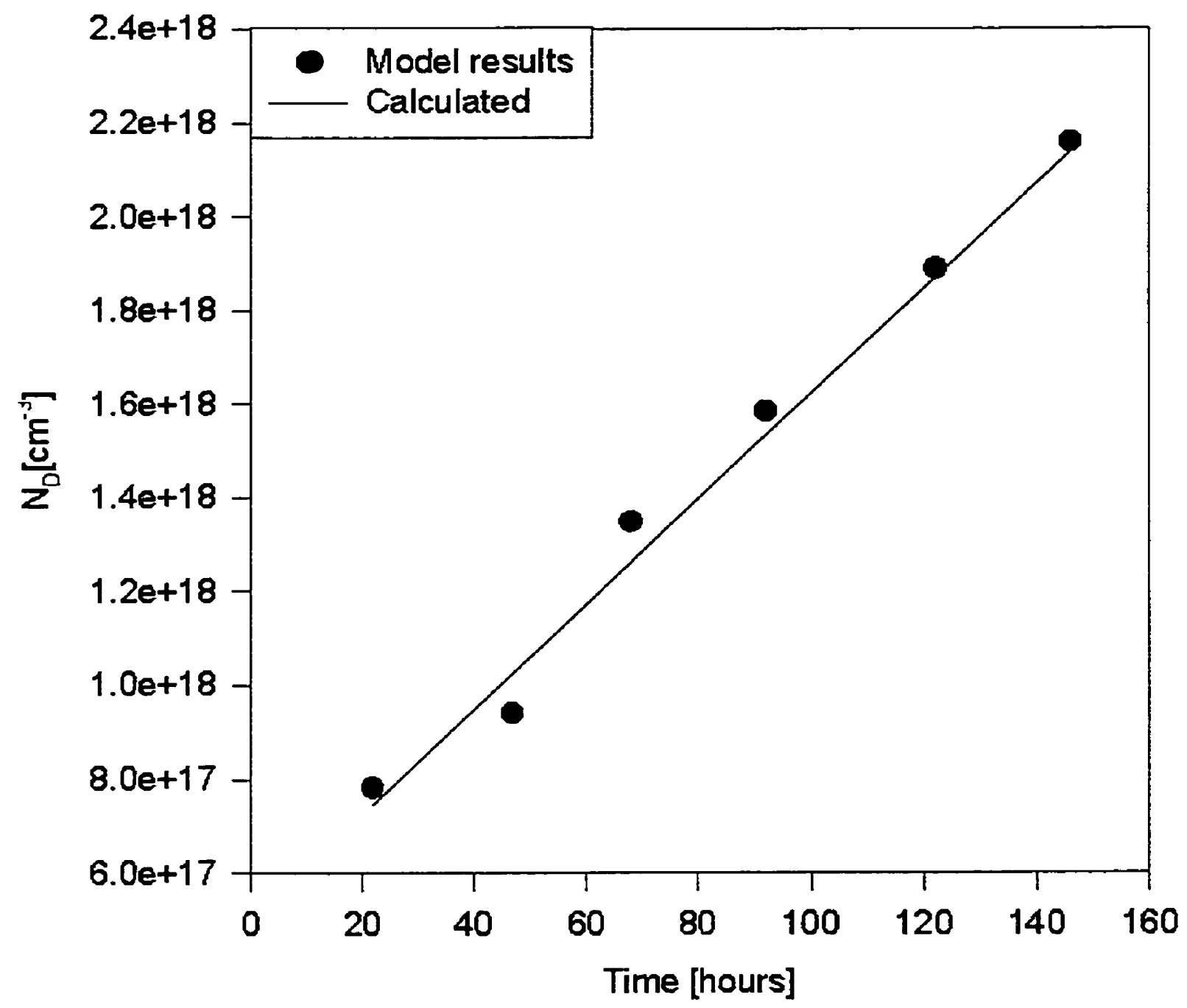

Figure 5.2. Time evolution of the total dangling bond concentration. and the fitted curve (6.5\% error) 
Mott estimates a value for the hopping distance of $R=30.5 \AA$ [6]. The results for the hopping distance calculated using the model are presented in figure 4.11. One can see that the values are similar to literature value.

The hopping distance is seen to increase with time. This can be explained by the shift in the Fermi energy. As the Fermi energy moves lower into the band-gap, the occupancy function for electrons will shift accordingly. The electrons will occupy states with lower energy in the conduction band tail, where the density of states is reduced. Therefore, the states available for hopping will be at larger distance than before and the hopping distance will increase, as can be seen in figure 4.11 . 


\section{Conclusions}

Tritiated amorphous silicon incorporates tritium in a stable bond with silicon. Tritium decays into helium, which diffuses away and leaves dangling bonds behind. These dangling bonds act as recombination centers.

Experimental results (from earlier work of Mr. Tome Kosteski and Dr. Franco Gaspari) show that the electrical conductivity of tritiated amorphous silicon film decreases with time. This can be explained by the increase in the concentration of the recombination centers. In this thesis, the time evolution of the electrical conductivity was used for the first time for a quantitative evaluation of the dangling bond concentration.

A model was developed to fit the experimental data. It considers thermally activated conductivity in the extended states, hopping conductivity in the conduction band tail states and beta conductivity in the extended states. The model for beta conductivity includes the concentration of the recombination centers, which is determined by the concentration of the dangling bonds.

The results of the fitting process show that the concentration of the neutrally charged dangling bonds $\left(\mathrm{D}^{0}\right.$ centers) increases with time. The concentration of the positively charged dangling bonds ( $\mathrm{D}^{+}$centers) decreases very slightly. This suggests that mainly $D^{0}$ centers act as recombination centers. 
The shift in the Fermi energy and the increase in the hopping distance are consistent with the increase in the density of $D^{0}$ centers with time.

The time evolution of the total concentration of the dangling bonds is in agreement with the nuclear decay of the tritium. This suggests that the dangling bonds are formed mainly at the sites of the decaying tritium atoms and allows a prediction of the dangling bond concentration at any ime after deposition. The model can be also used to calculate the initial dangling bond concentration in the sample and the initial atomic concentration of tritium using conductivity measurements. 


\section{References}

[1] R. A. Street, Hydrogenated Amorphous Silicon (Cambridge University Press, 1991).

[2] A. Madan and M.P. Shaw, The Physics and Applications of Amorphous Semiconductors (Academic Press, 1988).

[3] W. Fuhs, Electronic Properties of Amorphous Silicon in Amorphous \& Microcrystalline Semiconductor Devices, editor J. Kanicki (Artech House, 1992).

[4] W. Luft and Y.S. Tsuo, Hydrogenated Amorphous Silicon Alloy Deposition Processes (Marcel Dekker, 1993).

[5] Topics in Applied Physics 55 and 56, editors J. D. Joannopoulos and G. Lucovsky (Springer-Verlag 1984)

[6] N. F. Mott and E. A. Davis, Electronic Processes in Non-crystalline Materials (Clarendon Press, 1979).

[7] G. Bruno, P. Capezzuto and A. Madan, Plasma Deposition of Amorphous Silicon Based Materials (Academic Press, 1995).

[8] N. P. Kherani, $\mathrm{PhD}$. Thesis (University of Toronto, 1993)

[9] T. Kosteski, N.P. Kherani, F. Gaspari, S. Zukotynski, and W.T. Shmayda, J. Vac. Sci. Technol. A 16, 893 (1998).

[10] R.S. Crandall, Phys. Rev. B., 43, 4057 (1991).

[11] D. S. Shen, S. Wagner, J. Appl. Phys. 78, 278 (1995)

[12] H. Okamoto, Y. Hamakawa, Solid St. Commun. 24, 23 (1977) 
[13] D. Adler, E. J. Yoffa, Phys. Rev. Lett. 36, 11 (1976)

[14] T. J. McMahon, J. P. Xi, Phys. Rev. B 34, 4 (1986)

[15] J. A. Schmidt, R. D. Arce, R. R. Koropecki, R. H. Buitrago, Phys. Rev. B 59, 7 (1999)

[16] L. S. Sidhu, PhD. Thesis (University of Toronto, 1997) 